\title{
Natural Foods and Indian herbs of cardiovascular interest
}

\begin{abstract}
Since the start of human development, herbs have been an essential piece of society, esteemed for both their culinary and medicinal properties. Cardiovascular malady is the main executioner in the United States. Diet and way of life assume an imperative job in avoiding and switching heart infection, and certain herbs and supplements can help in bringing down hazard for heart ailment and treat heart conditions. Contrasted and customary prescriptions, herbal drugs don't require clinical investigations previously their showcasing or formal endorsement from administrative organizations, and thus their viability and wellbeing are once in a while demonstrated. Herba medications have been utilized in patients with congestive heart disappointment, systolic hypertension, angina pectoris, atherosclerosis, cerebral inadequacy, venous deficiency, and arrhythmia, CHF, since hundreds of years. Be that as it may, numerous herbal cures utilized today have not experienced cautious logical appraisal, and some can possibly make genuine lethal impacts and real medication tranquilize cooperation's. In spite of the greater part of these herbs demonstrating an impact on organic components identified with the cardiovascular system, information on their clinical impacts are inadequate. Conceivably applicable reactions, including expanded danger of medication connections, are portrayed, and the likelihood of tainting or substitution with different meds speaks to a worry. Doctors ought to dependably evaluate the utilization of herbal meds with patients and talk about the conceivable advantages and symptoms with them. Multidisciplinary explore is as yet required to misuse the tremendous capability of these plants. Potential synergistic and unfriendly reactions of herb-medicate communications additionally should be considered. These methodologies will help in setting up them as solutions for cardiovascular sicknesses and incorporating them in the standard of healthcare system. In spite of the fact that it has for quite some time been demonstrated that nutrient $\mathrm{D}$, ascorbic acid, and nutrient B12 are the way to treating unending ailments, including CVDs. Various regular carotenoids are available in new foods grown from the ground, and some have been concentrated widely in the counteractive action of coronary heart illness (CHD).
\end{abstract}

Volume 7 Issue 2 - 2019

\author{
AK Mohiuddin \\ Assistant Professor, Department of Pharmacy, World University \\ of Bangladesh, Bangladesh
}

Correspondence: AK Mohiuddin, Assistant Professor, Department of Pharmacy, World University of Bangladesh, 151/8, Green Road, Dhanmondi, Dhaka-1205, Bangladesh, Tel +8801716477485, Email trymohi@gmail.com

Received: February 20, 2019 | Published: March 22, 2019

Keywords: anti-oxidant, hypertension, cholesterol, atherosclerosis, cardioprotective, stroke

\section{Introduction}

The utilization of herbal medicine has soar in the course of the most recent 10 years, with wellbeing consumption assessed over $18 \%$ of the GDP in US in 2018 (Figure 1). In the traditional Indian system of medicine Ayurveda and Siddha different spices and herbs are portrayed to have medicinal properties, for example, being antithrombotic, hostile to atherosclerotic, hypolipidemic, hypoglycemic, calming, antiarthritic, and so forth. It has been accepted for quite a while that dietary factors assume a key job in the advancement of some human maladies, including cardiovascular sickness. A few herbs and spices of culinary origin were incorporated into the "endorsed" monographs, for example, caraway oil and seed, cardamom seed, cinnamon bark, cloves, coriander seed, dill seed, fennel oil and seed, garlic, ginger root, licorice root, mint oil, onion, paprika, parsley herb and root, peppermint leaf and oil, rosemary, sage, thyme, turmeric root, and white mustard seed. Spices are wealthy in cancer prevention agents, and a logical report recommends they are likewise strong inhibitors of tissue harm and irritation brought about by elevated amounts of glucose and coursing lipids. Aside from the treatment of cardiovascular hazard factors with pharmacological specialists and the utilization of antithrombotic drugs, there is developing attention to the job of dietary factors and herbal medicines in the anticipation of CVD and the likelihood of their utilization in treatment. Quite a bit of this intrigue focuses on the utilization of cancer prevention agent nutrients and the cell reinforcement properties of herbal materials, albeit some herbal materials may likewise enhance regular cardiovascular hazard factors or have antithrombotic impacts. Herb- tranquilize cooperation's are probably going to be increasingly genuine with medications having a thin remedial file, for example, warfarin or digoxin. The utilization of supplements of essential micronutrients (EMNs) in universal medicinal practice stays questionable, albeit sufficient measures of these substances are known to be important for the upkeep of wellbeing like Carotenoids, Vitamins B, C, D, E and K, Flavonoids, Magnesium and Iron, L-Carnitine, Omega-3 Polyunsaturated Fatty Acids, Coenzyme Q10. Different advantageous cardiovascular impacts have been found with garlic, Danshen, Lingzhi, Maidenhair Tree, Foxglove, Ginseng and so on. The proof to help the utilization of these elective treatments from clinical preliminaries isn't yet verify, yet custom and practice make it likely that they will keep on being utilized for the aversion or treatment of CVDs, among different signs. Current patterns for heftiness the executives include numerous pharmacological procedures, including blocking supplement assimilation, adjusting fat digestion, directing fat flags, and balancing the satiety focus, additionally exists in the extent of cardiac administration. Dietary phytochemicals have as of late stimulated significant enthusiasm as the potential helpful operators for wellbeing advancement and to balance stoutness. Because of their 
substance assorted variety and capacity to follow up on different organic targets, plant items have for some time been a flourishing hotspot for the revelation of new medications, and these discover use among the most widely recognized reciprocal and elective medicine systems (Figure 2).

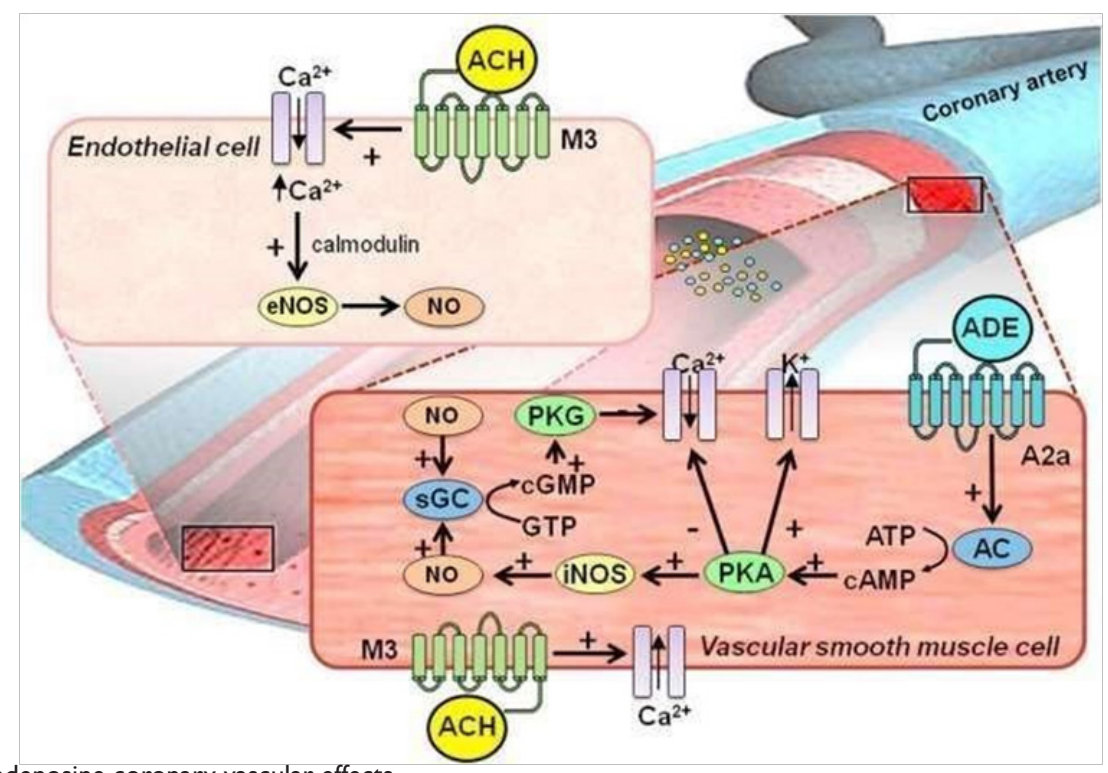

Figure I Acetylcholine and adenosine coronary vascular effects.

Acetylcholine and Adenosine Coronary Vascular Effects Acetylcholine $(\mathrm{ACH})$ has double contradictory impacts on the coronary veins. By authoritative to muscarinic 3 (M3) receptors on the outside of vascular smooth muscle cells, it inspires (bolt and + ) an intracellular arrival of calcium particles $\left(\mathrm{Ca}^{2+}\right)$, prompting vasoconstriction, while endothelial M3 receptor- intervened $\mathrm{Ca}^{2+}$ discharge enacts the endothelial nitric oxide (NO) synthase (eNOS, NOS III) through a calmodulin-subordinate pathway. NO is then discharged and, in vascular smooth muscle cells, actuates dissolvable guanylate-cyclase (sGC), which changes over (bended bolt) guanosine triphosphate (GTP) into cyclic guanosine monophosphate (cGMP). The resulting actuation of GMP-subordinate protein kinase (PKG) initiates a course of intracellular occasions with the last impact of diminishing (bolt and - ) intracellular $\mathrm{Ca}^{2+}$ fixations, prompting vasodilation. Adenosine (ADE) ties to its receptors (A2a) on the outside of vascular smooth muscle cells, initiating adenylate cyclase (AC) and prompting an expansion in cyclic adenosine monophosphate (CAMP) focus and cAMP-subordinate protein kinase (PKA) enactment. The last outcomes in potassium $\left(\mathrm{K}^{+}\right)$channel opening, bringing about a hyperpolarization of vascular smooth muscle cells, hinders the passage of $\mathrm{Ca}^{2+}$ and furthermore initiates inducible NOS (iNOS) (NOS II), hence delivering vasodilation. ATP $=$ adenosine triphosphate

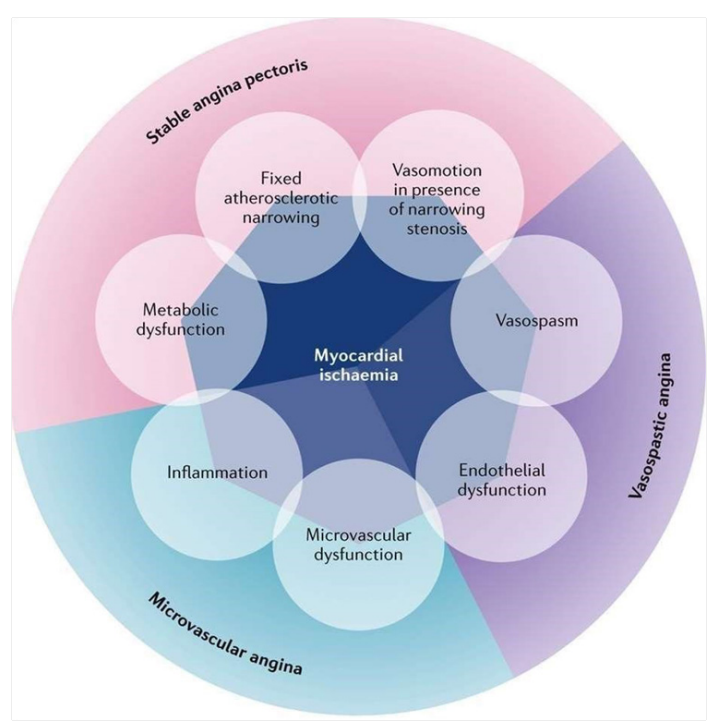

Figure 2 Different manifestations of myocardial ischemia.

Stable angina occurs when myocardial ischemia is caused by fixed atherosclerotic narrowing of one or more epicardial coronary arteries. In some circumstances, the angina is associated with a coronary spasm and metabolic dysfunction. Vasospastic angina occurs when myocardial ischemia is caused by a coronary artery spasm with or without endothelial dysfunction. Microvascular angina refers to the absence of an obstructed epicardial coronary artery. Myocardial ischemia in this case can be caused by microvascular and/or endothelial dysfunction and inflammation.

\section{Heart disease and significance}

Cardiovascular diseases include the blood vessels, the heart, or both. ${ }^{1}$ These incorporate various issues, a significant number of which are identified with a procedure called atherosclerosis. Atherosclerosis is a condition that creates when a substance called plaque develops in the dividers of the conduits. ${ }^{2}$ Arrhythmia alludes to a strange heart beat. ${ }^{3}$ There are different kinds of arrhythmias. The heart can pulsate excessively moderate, excessively quick or sporadically. At the point when heart valves don't open enough to enable the blood to course through as it should, a condition called stenosis results. At the point when the heart valves don't close legitimately and consequently enable blood to spill through, it's called spewing forth. On the off chance that the valve flyers lump or prolapse again into the upper chamber, it's a condition called prolapse. ${ }^{4,5}$ Hypertension is another name for hypertension. It can prompt serious confusions and expands the danger of heart malady, stroke, and demise. Circulatory strain is the power applied by the blood against the dividers of the veins. ${ }^{6}$ Myocardial ischemia happens when blood stream to heart is diminished, keeping it from getting enough oxygen. The diminished blood stream is normally the consequence of a fractional or complete blockage of heart's conduits (coronary courses). ${ }^{7}$

Traditional hazard factors for CVD incorporate more established age, smoking, hypertension, being overweight or large, diabetes, elevated cholesterol, and a family ancestry of heart sickness. ${ }^{8}$ Instances of other "nontraditional" chance factors that are once in a while utilized for hazard evaluation incorporate the lower leg brachial file (ABI), high-affectability C-responsive protein (hsCRP) level, and 
the coronary corridor calcium (CAC) score. The ABI is determined by contrasting circulatory strain esteems estimated at the lower leg and the arm (brachial supply route). High-affectability CRP is a protein associated with aggravation that is estimated by its dimension in an individual's blood. The CAC score estimates the measure of calcium in the veins of the heart dependent on a figured tomographic sweep of the chest (Figure 3) (Table 1). ${ }^{9-35}$

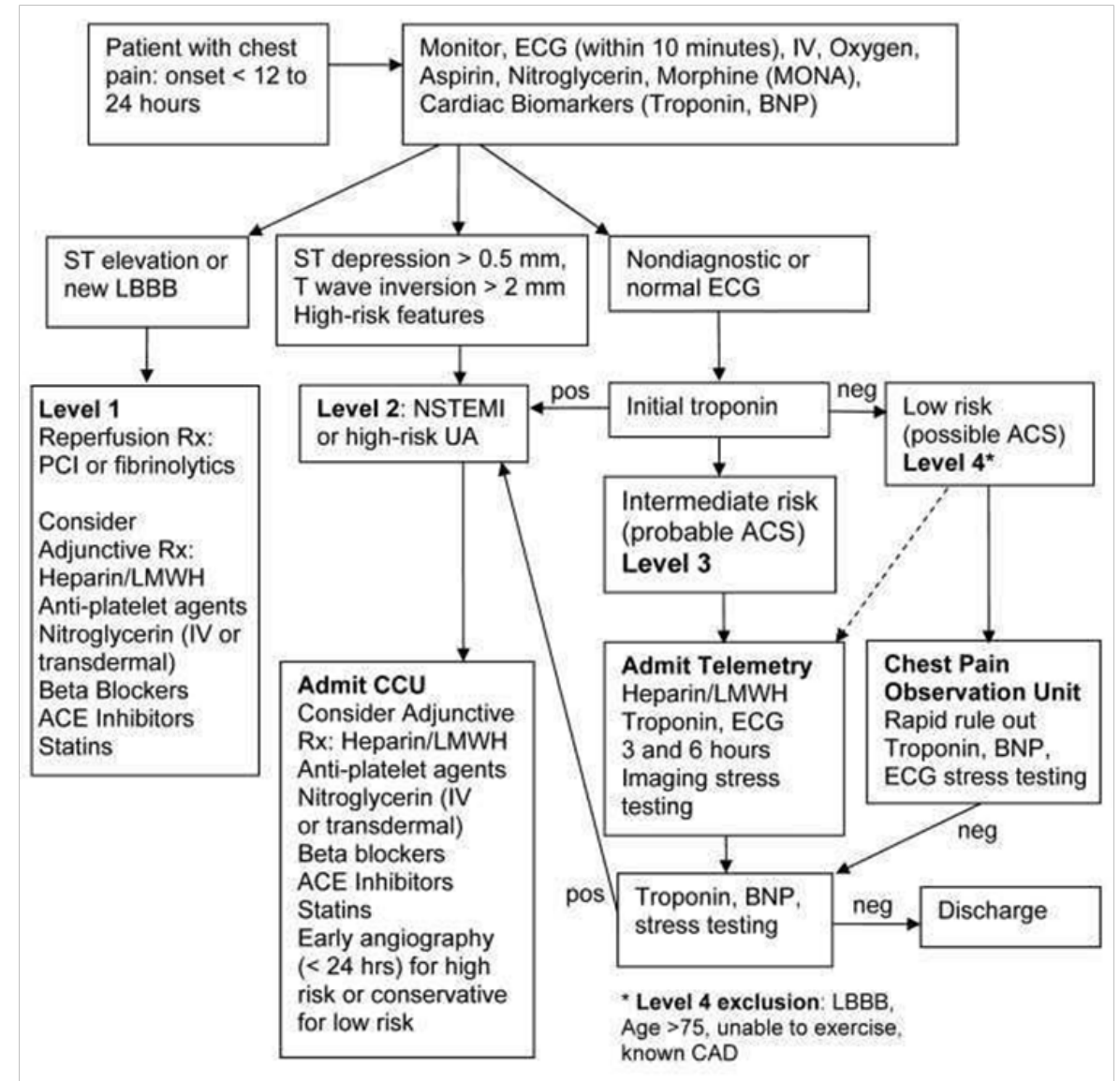

Figure 3 Chest pain evaluation algorithm.

An algorithm that integrates information from the above clinical decision rules with ECG findings. Low-risk patients are unlikely to have chest pain resulting from acute or chronic cardiac disease, although other serious causes (e.g., anxiety disorder, reflux disease, peptic ulcer, and pulmonary embolism) should be considered. Patients at high risk of CAD require urgent evaluation and, in many cases, hospitalization. For patients at moderate risk, ECG and clinical findings can be used to identify those who are at high or low risk. Cardiac troponin testing can be used for risk stratification if it is available (e.g., in urgent care settings). A normal troponin level at least six hours after the onset of chest pain in combination with normal or near-normal ECG findings is a good prognostic sign; only one in 300 patients with this combination of findings have a cardiovascular event within 30 days. 9 Although this algorithm has not been prospectively validated, it is based on prospectively validated diagnostic data.

Table I Complications of heart disease ${ }^{14-25}$

Heart failure

Heart attack

Stroke

Aneurysm

Peripheral artery disease

Sudden cardiac arrest
One of the most common complications of heart disease, heart failure occurs when heart can't pump enough blood to meet body's needs. Heart failure can result from many forms of heart disease, including heart defects, cardiovascular disease, valvular heart disease, heart infections or cardiomyopathy.

A blood clot blocking the blood flow through a blood vessel that feeds the heart causes a heart attack, possibly damaging or destroying a part of the heart muscle. Atherosclerosis can cause a heart attack.

The risk factors that lead to cardiovascular disease also can lead to an ischemic stroke, which happens when the arteries to brain are narrowed or blocked so that too little blood reaches brain. A stroke is a medical emergency-brain tissue begins to die within just a few minutes of a stroke.

A serious complication that can occur anywhere in your body, an aneurysm is a bulge in the wall of your artery. If an aneurysm bursts, you may face life threatening internal bleeding.

Atherosclerosis also can lead to peripheral artery disease. When peripheral artery disease is developed in extremities, usually legs don't receive enough blood flow. This causes symptoms, most notably leg pain when walking (claudication).

Sudden cardiac arrest is the sudden, unexpected loss of heart function, breathing and consciousness, often caused by an arrhythmia. Sudden cardiac arrest is a medical emergency. If not treated immediately, it is fatal, resulting in sudden cardiac death. 
In 2011, the AHA made another arrangement of vital Impact Goals not exclusively to diminish CVD passings, yet additionally to enhance cardiovascular wellbeing, made out of 7 measurements (Life's Simple
7). These incorporate 4 wellbeing practices (diet, physical movement, smoking, and weight list) and 3 wellbeing factors (blood cholesterol, circulatory strain, and blood glucose) (Table 2). ${ }^{36-38}$

Table 2 AHA's life's simple 7 CVH Score ${ }^{36}$

\begin{tabular}{|c|c|c|c|}
\hline CVH Metric & Ideal CVH definition (2 Points) & Intermediate CVH definition (I Point) & Poor CVH definition (0 Point) \\
\hline Smoking & Never smoker & Former smoker & Current smoker \\
\hline Body mass index, $\mathrm{kg} / \mathrm{m}^{2}$ & $<25$ & $25-29.9$ & $>30$ \\
\hline Physical activity & $\begin{array}{l}\geq 150 \mathrm{~min} / \mathrm{wk} \text { moderate or } \\
\geq 75 \mathrm{~min} / \mathrm{wk} \text { vigorous or } \\
\geq 150 \mathrm{~min} / \mathrm{wk} \\
\text { moderate+vigorous activity }\end{array}$ & $\begin{array}{l}\text { I-149 min/wk moderate or I- } \\
74 \mathrm{~min} / \mathrm{wk} \text { vigorous or I- } \\
149 \mathrm{~min} / \mathrm{wk} \text { moderate+vigorous activity }\end{array}$ & None \\
\hline Diet score, no. of components ${ }^{a}$ & $4-5$ & $2-3$ & $0-1$ \\
\hline Total cholesterol, mg/dL & $<200^{b}$ & $200-239^{\circ}$ or treated to goal & $\geq 240$ \\
\hline Blood pressure & $<120 /<80 \mathrm{~mm} \mathrm{Hg}^{\mathrm{b}}$ & $\begin{array}{l}\mathrm{SBP} \text { I } 20-139 \mathrm{~mm} \mathrm{Hg}^{\mathrm{b}} \text { and/or DBP } 80-89 \\
\mathrm{~mm} \mathrm{Hg} \text { or treated to }<120 /<80 \mathrm{~mm} \mathrm{Hg}\end{array}$ & $\begin{array}{l}\mathrm{SBP} \geq 140 \mathrm{~mm} \mathrm{Hg} \text { and/or DBP } \\
\geq 90 \mathrm{~mm} \mathrm{Hg}\end{array}$ \\
\hline Fasting glucose, $\mathrm{mg} / \mathrm{dL}^{\mathrm{b}}$ & $<100$ & $100-125$ & $\geq 126$ \\
\hline
\end{tabular}

aFruits and vegetables: $\geq 4.5$ cups/d; fish: $\geq 23.5$-oz servings/wk (preferably oily fish); fiber-rich whole grains ( $\geq \mathrm{I}$.I $\mathrm{g}$ of fiber per $10 \mathrm{~g}$ of carbohydrate): $\geq 3 \quad \mathrm{I}-\mathrm{oz}$ equivalent servings/d; sodium: <1500 mg/d; sugar sweetened beverages: $\leq 450 \mathrm{kcal}$ (36 oz)/wk. bUntreated values.

\section{Prevalence and economic burden of cardiac diseases}

In 2014, US future positioned 43rd on the planet, despite the fact that the United States spent the most (\$3.0 trillion) on human services, surpassing the middle sum spent by Organization for Economic Coactivity and Development nations by $35 \% .{ }^{39} \mathrm{CVD}$ was the main source of death in the United States in 2016, representing more than 900000 deaths. Most CVD trouble in the United States is from atherosclerotic vascular infection, and $80 \%$ can be ascribed to realized causal hazard factors. ${ }^{30}$ Almost 1 of every 3 (around 80 million) grown-ups have some type of CVD, which confers a substantial financial weight, including assessed direct expenses of roughly $\$ 444$ billion in US. The CVD expenses of consideration are proceeding to ascend, with the present expenses for treatment representing almost $\$ 1$ of each $\$ 6$ spent on social insurance. ${ }^{40}$ The proof on the monetary weight of CVD in LMICs stays rare. The expenses per scene for hypertension and nonexclusive CVD were genuinely homogeneous crosswise over investigations; extending somewhere in the range of $\$ 500$ and $\$ 1500$. Interestingly, for CHD and stroke cost assessments were commonly higher and progressively heterogeneous, with a few gauges in overabundance of $\$ 5000$ per scene. Normal month to month treatment costs for stroke and CHD extended somewhere in the range of $\$ 300$ and $\$ 1000$ in China, Brazil, India and Mexico. ${ }^{41}$

Predominance of affirmed hypertension in youngsters to run somewhere in the range of $2 \%$ and $4 \% .{ }^{26}$ In a few nations, up to $75 \%$ of more established grown-ups are hypertensive. ${ }^{27} 87 \%$ of the grownups in the US who self-revealed having hypertension take part in at least two exercises to diminish pulse, for example, taking prescription, participating in physical action, evolving diet, and decreasing liquor utilization. $^{28}$ In November 2017, new rules from the American College of Cardiology and the American Heart Association (ACC/ AHA) extended the meaning of hypertension, stretching out the mark to $46 \%$ of grown-ups in the United States. ${ }^{31}$ CHD is a noteworthy reason for death and handicap in created nations. Despite the fact that the mortality for this condition has slowly declined in the course of the most recent decades in western nations, regardless it causes around $33 \%$ of all passings in individuals more seasoned than 35 years. ${ }^{38}$ Atrial fibrillation is the most well-known continued arrhythmia, increments with age, and gives a wide range of manifestations and seriousness. ${ }^{32}$ The latest investigations have affirmed this discernment and demonstrated that the predominance of AF in the general grownup populace of Europe is more than twofold that detailed only multi decade sooner, extending from $1.9 \%$ in Italy, Iceland, and England to $2.3 \%$ in Germany and $2.9 \%$ in Sweden. ${ }^{33}$ The assessed commonness is lower in ladies (373 for every 100,000) than in men (596 for each $100,000)$

AF frequency has been appeared to increment lopsidedly with expanding age in the two ladies and men, coming to as high as 30.4 per 1000 man a very long time in ladies and 32.9 per 1000 man a long time in men by age $85-89$ years. ${ }^{34}$ Most investigations of intense myocardial localized necrosis (AMI) the study of disease transmission and treatment have concentrated on patients who experience the beginning of AMI outside of the emergency clinic. It is progressively perceived that AMI additionally happens among patients previously hospitalized for different conditions. ${ }^{35}$ All inclusive, stroke is the second driving reason for death and third driving reason for disability. 1 More than $74 \%$ of the weight of stroke has been ascribed to smoking, less than stellar eating routine, and low physical movement, while over $72 \%$ has been credited to metabolic hazard factors (high plasma glucose, elevated cholesterol, hypertension, overweight and heftiness, and kidney ailment). ${ }^{37}$ Myocardial areas of localized necrosis (MIs) are among the main sources of dismalness and mortality in the United States and lead to $>\$ 11$ billion in yearly hospitalization costs. Of people $>45$ years old who have a first MI, frequency of repetitive MI or deadly coronary heart illness inside 5 years extents from $17 \%$ to $20 \%$, and heart disappointment rates are comparable, including further healthcare costs, which are anticipated to increment by practically $100 \%$ by $2030 .{ }^{36}$ 


\section{Nutritional recommendations cardiovascular disease prevention}

Cardiometabolic illnesses are evaluated to cause more than 700,000 passings for every year in the US and about half of these passings are straightforwardly identified with eating routine. ${ }^{42}$ Way of life factors, including nourishment, assume a critical job in the etiology of CVD. CVD hazard in postmenopausal ladies seems, by all accounts, to be delicate to a change to a low-fat dietary example and, among sound ladies, incorporates both CHD advantage and stroke chance. ${ }^{43}$ Factors that impact people to devour a low-quality eating regimen are heap and incorporate absence of information, absence of accessibility, staggering expense, time shortage, social and social standards, promoting of low quality nourishments, and attractiveness. ${ }^{44}$ The medicinal writing is still brimming with articles contending contradicting positions. For instance, in 2017, after an audit of the proof, the AHA Presidential Advisory firmly embraced that "bringing down admission of immersed fat and supplanting it with unsaturated fats, particularly polyunsaturated fats, will bring down the rate of CVD". After three months, the 18-nation observational Prospective Rural Urban Epidemiology (PURE) Study finished up much the inverse: "Absolute fat and kinds of fat were not related with cardiovascular sickness, myocardial dead tissue, or cardiovascular illness mortality". ${ }^{45}$

Today, there keeps on being an enthusiasm for low-carb methodologies, for example, Atkins, Banting, ketogenic, and South Beach. While eats less carbs instigating weight reduction delivers a caloric shortage, the system of low-carb slims down stays in discussion. When bringing down starches from the eating regimen, the macronutrient admission of fat and protein by and large increments to make up for the decrease of sugars. One speculation of why lowcarb approaches produces quick weight reduction contrasted with different eating regimens is that fats and protein increment satiety and produce less attendant hypoglycemia. This expansion in satiety and less bounce back hypoglycemia at that point decreases yearning and by and large nourishment admission and produces a caloric deficiency. ${ }^{46}$ Both high and low rates of sugar consumes less calories were related with expanded mortality, with insignificant hazard saw at $50-55 \%$ starch admission. Plant-inferred protein and fat admission, from sources, for example, vegetables, nuts, nutty spread, and entire grain breads, were related with lower mortality, proposing that the wellspring of sustenance quite adjusts the relationship between starch admission and mortality. ${ }^{47}$ The traditional Mediterranean eating routine is portrayed by the utilization of entire grains, vegetables, natural products, vegetables, nuts, fish and olive oil, wine with some restraint, and a moderate admission of meat, dairy items, prepared sustenance's and desserts. ${ }^{48}$

Mediterranean eating routine decreased cardiovascular ailment mortality chance identified with long haul introduction to air contaminations in a vast forthcoming U.S partner. Expanded utilization of nourishments wealthy in cancer prevention agent compounds may help in decreasing the extensive ailment trouble related with encompassing air contamination. ${ }^{49}$ As appeared in a metaexamination of seven partner thinks about; a 2-point increment in adherence to the Mediterranean eating regimen was related with a noteworthy decrease of in general mortality. ${ }^{50}$ Furthermore, a meta-examination of 23 forthcoming accomplice investigations of 937,665 members and 18,047 CHD patients demonstrated that natural product utilization was contrarily connected with a danger of CHD. ${ }^{51}$ Second rate aggravation, instead of lipids, is probably going to be on the pathway of the communication among MD and statins towards mortality chance. MD brought down the danger of all-cause, cardiovascular and CAD/cerebrovascular mortality CVD patients, net of statins (Table 3)..$^{52}$

Table 3 Recommendations of dietary patterns in prevention of CVD ${ }^{50}$

\begin{tabular}{ll}
\hline Food pattern & Recommendations \\
\hline Low-fat diet & $\begin{array}{l}\text { Low-fat diet with restricted calories may present a healthy alternative to the typical Western diet. It may improve quality and life } \\
\text { expectancy in healthy people, as well as in patients with overweight, diabetes, and CVD. }\end{array}$ \\
& $\begin{array}{l}\text { In the short-run, low-carbohydrate diets lead to a greater weight loss compared to low-fat diets. Some studies have shown that } \\
\text { this advantage is retained at } 2 \text { years but not at longer follow-up periods }\end{array}$ \\
Low-carbohydrate diets are preferable to a low-fat diet in reducing TG levels and increasing HDL-C blood levels. It should be \\
emphasized that carbohydrates should preferably be replaced by unsaturated vegetable fats. \\
Low-carbohydrate diets, which include $30 \%-40 \%$ of calories from carbohydrates and are low in saturated fat and high in \\
monounsaturated fat, were found to be safe in healthy and overweight individuals at follow-up up to 4 years.
\end{tabular}

A Mediterranean diet with restricted calories may present a healthy alternative to the typical Western diet. It may improve quality and life expectancy in healthy people, as well as in patients with overweight, diabetes, and CVD.

Mediterranean Diet Mediterranean diets are preferable to a low-fat diet in reducing TG levels, increasing HDL-C blood levels, and improving insulin sensitivity.

DASH Diet

The DASH diet is recommended to prevent hypertension and lower blood pressure. The diet should be accompanied by lifestyle changes such as: weight reduction in overweight people, increased physical activity, sodium restriction, and alcohol avoidance.

\section{Natural foods as means of cardio-preventive measures}

Contrasted with refined grains, entire grains are higher in fiber, which may help diminish "terrible" LDL cholesterol and reduction the danger of heart infection. ${ }^{53,54}$ Berries are likewise wealthy in cell reinforcements like anthocyanins, which ensure against the oxidative pressure and irritation that add to the advancement of heart illness. ${ }^{55}$
Avocados are an incredible wellspring of heart solid monounsaturated fats, which have been connected to diminished dimensions of cholesterol and a lower danger of heart infection. ${ }^{56}$ Walnuts are an extraordinary wellspring of fiber and micronutrients like magnesium, copper and manganese. Of the unsaturated fats, oleic and linoleic acids speak to the greater part of the all out fat substance in pistachios. Pistachios are additionally a decent wellspring of vegetable protein (about $21 \%$ of all out weight), with an essential amino acid proportion 
higher than most other regularly devoured nuts (ie, almonds, walnuts, pecans, and hazelnuts), and they have a high level of expanded chain amino acids. ${ }^{57}$ Dull chocolate is wealthy in cancer prevention agents like flavonoids, which can help support heart wellbeing. ${ }^{58}$ Tomatoes are stacked with lycopene, a characteristic plant color with amazing cancer prevention agent properties. ${ }^{59}$ Almonds are likewise a decent wellspring of heart-solid monounsaturated fats and fiber, two essential supplements that can help secure against heart illness (Table 4). ${ }^{60}$

Table 4 The cardioprotective abilities of fruits ${ }^{51}$

\begin{tabular}{|c|c|c|c|c|}
\hline Fruit & Subject & $\begin{array}{l}\text { Study } \\
\text { Type }\end{array}$ & Dose & Main Effects \\
\hline $\begin{array}{l}\text { freeze-dried grape } \\
\text { powder }\end{array}$ & $\begin{array}{l}\text { SHR and Wistar- } \\
\text { Kyoto (WKY) rats }\end{array}$ & in vivo & $600 \mathrm{mg} /$ day & $\begin{array}{l}\mathrm{BP} \downarrow \text {, arterial relaxation } \uparrow \text {, vascular compliance } \uparrow \text {, cardiac } \\
\text { hypertrophy } \downarrow\end{array}$ \\
\hline GSPE & SHR & in vivo & $250 \mathrm{mg} / \mathrm{kg} /$ day & arterial remodeling $\downarrow, \mathrm{ET}-\mathrm{I} \downarrow, \mathrm{NO} \uparrow, \mathrm{SOD} \uparrow, \mathrm{CAT} \uparrow, \mathrm{MDA} \downarrow$ \\
\hline GP-EE & $\begin{array}{l}\text { rat aorta and small } \\
\text { mesenteric artery } \\
\text { (SMA) segments }\end{array}$ & in vitro & 0.3 and $10 \mu \mathrm{M}$ & $\begin{array}{l}\text { endothelium- and NO-dependent vasodilatation } \uparrow \text {, } \\
\text { phenylephrine(Phe)-induced response in aortic rings } \downarrow, O_{2}-\downarrow \text {, } \\
\text { contraction elicited by ET-I } \downarrow\end{array}$ \\
\hline $\begin{array}{l}\text { red grape skin and } \\
\text { seeds polyphenols }\end{array}$ & $\begin{array}{l}\text { human endothelial } \\
\text { progenitor cells } \\
\text { (EPC) }\end{array}$ & in vitro & 5,50 and $150 \mu g / m L$ & $\begin{array}{l}\text { EPC viability and function } \uparrow \text {, endothelial dysfunction } \downarrow \text {, } \\
\text { hyperglycemia effect } \downarrow \text {, ROS production } \downarrow\end{array}$ \\
\hline GSPE & $\begin{array}{l}\text { ouabain induced } \\
\text { hypertensive rats } \\
\text { model }\end{array}$ & in vivo & $250 \mathrm{mg} / \mathrm{kg} /$ day & $\mathrm{BP} \downarrow$, aortic NO production $\uparrow$ \\
\hline GPE & $\begin{array}{l}\text { endothelial (EA. } \\
\text { hy926) cells }\end{array}$ & in vitro & 0.068 and $0.250 \mu g / m L$ & GCS levels $\uparrow$, GST activity $\uparrow$, antioxidant activity $\uparrow$ \\
\hline GSE & HUVECs & in vitro & $\mathrm{I} \mu \mathrm{g} / \mathrm{mL}$ & platelet reactivity $\downarrow$ \\
\hline \multirow{3}{*}{$\begin{array}{l}\text { red grape berry } \\
\text { powder }\end{array}$} & $\begin{array}{l}\text { rats with metabolic } \\
\text { syndrome }\end{array}$ & in vivo & $\begin{array}{l}200,400 \text { and } 800 \mathrm{mg} / \\
\mathrm{kg} / \text { day }\end{array}$ & BP $\downarrow$, plasma TG $\downarrow$, insulin $\downarrow$ \\
\hline & & & $20-1400 \mu g / m L$ & $\mathrm{ET}-\mathrm{I} \downarrow$ \\
\hline & HUVECs & in vitro & $\begin{array}{l}0.011,0.058,0.29,1.46 \\
\text { and } 3.66 \mathrm{mg} / \mathrm{mL}\end{array}$ & eNOS level $\uparrow$ \\
\hline $\begin{array}{l}\text { grape seed } \\
\text { procyanidin extract }\end{array}$ & hamster & in vivo & $25 \mathrm{mg} / \mathrm{kg} /$ day & $\begin{array}{l}\text { body weight gain } \downarrow \text {, adiposity index } \downarrow \text {, weight of white adipose } \\
\text { tissue depots } \downarrow \text {, plasma phospholipids } \downarrow \text {, plasma FFA } \downarrow \text {, mesenteric } \\
\text { lipid and triglyceride accumulation } \downarrow\end{array}$ \\
\hline $\begin{array}{l}\text { grape polyphenols } \\
\text { from Vitis vinifera } \\
\text { grapes }\end{array}$ & $\begin{array}{l}\text { 24-month-old obese } \\
\text { rats }\end{array}$ & in vivo & $90 \mathrm{mg} / \mathrm{kg} / \mathrm{day}$ & plasma HDL PON activity $\uparrow$, LCAT activity $\uparrow$, CETP activity $\downarrow$ \\
\hline $\begin{array}{l}\text { grape seed } \\
\text { procyanidin extract }\end{array}$ & SHR & in vivo & $375 \mathrm{mg} / \mathrm{kg}$ & $\mathrm{SBP} \downarrow, \mathrm{DBP} \downarrow, \mathrm{GSH}$ activity $\uparrow$ \\
\hline $\begin{array}{l}\text { GSE or black } \\
\text { chokeberry (Aronia } \\
\text { melanocarpa) } \\
\text { extract }\end{array}$ & $\begin{array}{l}\text { human platelets } \\
\text { incubated with Hcy } \\
(100 \mu \mathrm{M}) \text { or } \mathrm{HTL} \\
(\mathrm{I} \mu \mathrm{M})\end{array}$ & in vitro & $2.5,5,10 \mu \mathrm{g} / \mathrm{mL}$ & $\begin{array}{l}\text { platelet adhesion to collagen and fibrinogen } \downarrow \text {, platelet } \\
\text { aggregation } \downarrow, \mathrm{O}_{2}^{-}-\text {production in platelet } \downarrow\end{array}$ \\
\hline $\begin{array}{l}\text { malvidin-rich red } \\
\text { grape skin extract }\end{array}$ & $\begin{array}{l}\text { isolated and } \\
\text { Langendorff perfused } \\
\text { rat heart }\end{array}$ & in vitro & $\mathrm{I}-1000 \mathrm{ng} / \mathrm{mL}$ & $\begin{array}{l}\text { I/R damages } \downarrow \text {, coronary dilation } \uparrow \text {, active } \mathrm{PI} 3 \mathrm{~K} / \mathrm{NO} / \mathrm{cGMP} / \mathrm{PKG} \\
\text { pathway, intracellular cGMP } \uparrow, \mathrm{eNOS}, \mathrm{PI} 3 \mathrm{~K}-\mathrm{AKT}, \mathrm{ERK} \mathrm{I} / 2 \text {, and } \\
\text { GSK-3 } \beta \text { phosphorylation } \uparrow\end{array}$ \\
\hline GSSE & $\begin{array}{l}\text { a rat model of global } \\
\text { ischemia }\end{array}$ & in vivo & $2.5 \mathrm{~g} / \mathrm{kg}$ & $\begin{array}{l}\text { brain damage size and histology } \downarrow \text {, oxidative stress } \downarrow \text {, transition } \\
\text { metals associated enzyme activities } \uparrow\end{array}$ \\
\hline GSPE & isolated rat hearts & in vitro & NA & $\begin{array}{l}\mathrm{RA} \downarrow, \mathrm{Na}^{+} / \mathrm{K}^{+} \text {-ATPase activity } \uparrow, \mathrm{Na}^{+} / \mathrm{K}^{+} \text {-ATPase } \alpha \mathrm{I} \text { subunit } \uparrow \text {, free } \\
\text { radical } \downarrow\end{array}$ \\
\hline GSPE & $\begin{array}{l}\text { a rat model of deep } \\
\text { vein thrombosis } \\
\text { (DVT) }\end{array}$ & in vivo & $400 \mathrm{mg} / \mathrm{kg} /$ day & $\begin{array}{l}\text { thrombus length and weight } \downarrow \text {, protecte endothelium integrity, IL-6, } \\
\text { IL-8 and TNF- } \alpha \downarrow\end{array}$ \\
\hline $\begin{array}{l}\text { blueberry extract } \\
\text { (Vaccinium ashei } \\
\text { Reade) }\end{array}$ & $\begin{array}{l}\text { hypercholesterol } \\
\text { emic rat }\end{array}$ & in vivo & $25,50 \mathrm{mg} / \mathrm{kg}$ & $\begin{array}{l}\text { aortic lesions } \downarrow \text {, oxidative damage to lipids and proteins } \downarrow, T C \downarrow \text {, } \\
\text { LDL-C } \downarrow, T G \downarrow \text {, activity of CAT, SOD and GSH-Px } \uparrow\end{array}$ \\
\hline $\begin{array}{l}\text { freeze-dried } \\
\text { blueberry powder }\end{array}$ & $\begin{array}{l}\text { rats fed a high-fat/ } \\
\text { cholesterol diet }\end{array}$ & in vivo & $2 \%(w / w)$ & SBP $\downarrow$, aorta relaxation $\uparrow$, endothelial dysfunction $\downarrow$ \\
\hline
\end{tabular}


Table Continued....

\begin{tabular}{|c|c|c|c|c|}
\hline Fruit & Subject & $\begin{array}{l}\text { Study } \\
\text { Type }\end{array}$ & Dose & Main Effects \\
\hline $\begin{array}{l}7 \text { phenolic acids } \\
\text { of freeze-dried } \\
\text { blueberry }\end{array}$ & $\begin{array}{l}\text { murine macrophage } \\
\text { cell line RAW } 264.7\end{array}$ & in vitro & NA & $\begin{array}{l}\text { TNF- } \alpha \text { and IL- } 6 \text { mRNA expression and protein levels } \downarrow \text {, MAPK, } \\
\text { JNK, } 338 \text {, and Erk I/2 phosphorylation } \downarrow, \text { mRNA expression and } \\
\text { protein levels of scavenger receptor CD } 36 \downarrow \text {, foam cell formation } \downarrow \text {, } \\
\text { expression and protein levels of ABCAI } \uparrow\end{array}$ \\
\hline PE & $\begin{array}{l}\text { SR-BI/apoE double } \\
\text { KO mice }\end{array}$ & in vivo & $307.5 \mu \mathrm{L} / \mathrm{L}$ in water & $\begin{array}{l}\text { aortic sinus and coronary artery atherosclerosis } \downarrow \text {, oxidative stress } \\
\text { and inflammation in the vessel wall } \downarrow\end{array}$ \\
\hline $\begin{array}{l}\text { PE containing } 40 \% \\
\text { punicalagin }\end{array}$ & SHR & in vivo & 150 mg/kg/day & $\begin{array}{l}\text { BP } \downarrow \text {, cardiac hypertrophy } \downarrow \text {, oxidative stress } \downarrow \text {, antioxidant defense } \\
\text { system } \uparrow \text {, paraventricular nucleus inflammation } \downarrow \text {, mitochondrial } \\
\text { superoxide anion levels } \downarrow \text {, mitochondrial function } \uparrow\end{array}$ \\
\hline $\begin{array}{l}\text { PE containing } 40 \% \\
\text { punicalagin }\end{array}$ & $\begin{array}{l}\text { heart of a high-fat } \\
\text { diet-induced obesity } \\
\text { rat model }\end{array}$ & in vivo & 150 mg/kg/day & $\begin{array}{l}\text { mitochondrial biogenesis } \uparrow \text {, oxidative stress } \downarrow \text {, phase II enzymes } \uparrow \text {, } \\
\text { cardiac metabolic disorders } \downarrow\end{array}$ \\
\hline $\begin{array}{l}\text { Bravo de Esmolfe } \\
\text { apple }\end{array}$ & $\begin{array}{l}\text { male Wistar } \\
\text { rats fed a } \\
\text { cholesterolenriched } \\
\text { diet }(+2 \% \\
\text { cholesterol) }\end{array}$ & in vivo & $\begin{array}{l}20 \%(w / w)=5 g / r a t / \\
\text { day }(\sim 2-3 \text { apples/ } \\
\text { perso } n / \text { day }) \text { for } 30 \\
\text { days }\end{array}$ & serum TG $\downarrow, T C \downarrow$, LDL-C $\downarrow$, oxLDL $\downarrow$ \\
\hline $\begin{array}{l}\text { Fuji apple peel } \\
\text { Granny Smith } \\
\text { apple peel }\end{array}$ & $\begin{array}{l}\text { CF-I mice with } \mathrm{MS} \\
\text { apoE }{ }^{-l-} \text { mice }\end{array}$ & in vivo & $\begin{array}{l}20 \%(w / w) \text { for } 43 \text { days } \\
20 \%(w / w) \text { for } 10 \\
\text { weeks }\end{array}$ & $\begin{array}{l}\text { glycaemia } \downarrow, T C \downarrow, H D L-C \downarrow \text {, LDL-C } \downarrow \text {, ureic nitrogen } \downarrow, T G \downarrow \text {, insulin } \downarrow \text {, } \\
\text { ADMA } \downarrow \text { atherogenic progression } \downarrow \text {, cholesterol accumulation } \\
\text { area } \downarrow\end{array}$ \\
\hline HFC & $\begin{array}{l}\text { apoE }{ }^{-/-} \\
\text {atherosclerotic mice } \\
\text { with high blood lipid } \\
\text { levels fed with a high } \\
\text { cholesterol diet }\end{array}$ & in vivo & $0.5 \mathrm{~mL} /$ day & TG $\downarrow$, LDL-C/TC ratio $\downarrow$ \\
\hline HPPS & $\begin{array}{l}\text { the liver of high } \\
\text { fat diet induced } \\
\text { hyperlipidemic mice }\end{array}$ & in vivo & $150 \mathrm{mg} / \mathrm{kg}$ & $\begin{array}{l}\text { weight gain } \downarrow, T G \downarrow \text {, lipid excretion in feces } \uparrow \text {, mRNAs and activities } \\
\text { of acyl-CoA oxidase, carnitine palmitoyltransferase I, 3-ketoacyl- } \\
\text { CoA thiolase, and } 2,4 \text {-dienoyl-CoA reductase } \uparrow \text {, gene and protein } \\
\text { expressions of PPAR- } \alpha \uparrow\end{array}$ \\
\hline $\begin{array}{l}\text { freeze dried } \\
\text { hawthorn fruit } \\
\text { (Crataegus } \\
\text { pinnatifida) }\end{array}$ & $\mathrm{apoE}^{-/-}$mice & in vivo & $1 \%(w / w)$ & $\begin{array}{l}\text { atherosclerotic lesions } \downarrow, T C \downarrow, T G \downarrow, T-A O C \text { values } \uparrow, \text { SOD and } \\
\text { GSH-Px activities } \uparrow \text {, hepatic FAS and SREBP-Ic mRNA levels } \downarrow \text {, } \\
\text { hepatic SODI, SOD2, Gpx3 mRNA levels } \uparrow\end{array}$ \\
\hline $\begin{array}{l}\text { sugar-free } \\
\text { aqueous extract } \\
\text { of hawthorn } \\
\text { fruit (Crataegus } \\
\text { pinnatifida var. } \\
\text { Major) }\end{array}$ & high fat diet fed rats & in vivo & 72 and $288 \mathrm{mg} / \mathrm{kg} /$ day & $\begin{array}{l}\text { TC, TG and LDL-C } \downarrow, \mathrm{HDL}-\mathrm{C} \uparrow, \mathrm{CRP}, \mathrm{IL}-\mathrm{I} \beta, \mathrm{IL}-8 \text { and IL- } I 8 \downarrow, \mathrm{ET} \text {, } \\
\text { 6-keto-PGFI } \alpha \text { and TXB2 } \uparrow \text {, pathological changes in the arteries } \downarrow \text {, } \\
\mathrm{IMT} \downarrow\end{array}$ \\
\hline \multirow[t]{2}{*}{$\begin{array}{l}\text { avocado pulp } \\
\text { (Persea americana) } \\
\text { extract }\end{array}$} & $\begin{array}{l}\text { male adult } C D \text { I } \\
\text { mice }\end{array}$ & in vivo & $25 \mathrm{mg} / \mathrm{kg}$ & thrombus formation $\downarrow$ \\
\hline & platelet & in vitro & $10 \mu \mathrm{L}$ & platelet aggregation $\downarrow$ \\
\hline avocado oil & $\begin{array}{l}\text { rats ingested with } \\
\text { sucrose }\end{array}$ & in vivo & $7.5 \%(w / w)$ & TG $\downarrow, \mathrm{VLDL} \downarrow, \mathrm{LDL} \downarrow$, hs-CRP $\downarrow$ \\
\hline $\begin{array}{l}\text { freeze-dried mango } \\
\text { pulp }\end{array}$ & $\begin{array}{l}\text { male } \text { C57BL/6J mice } \\
\text { fed a highfat diet }\end{array}$ & in vivo & $1 \%$ or $10 \%(w / w)$ & $\begin{array}{l}\text { epididymal fat mass } \downarrow \text {, percentage of body fat } \downarrow \text {, improve glucose } \\
\text { tolerance, insulin resistance } \downarrow\end{array}$ \\
\hline $\begin{array}{l}\text { methanolic extract } \\
\text { of papaya (Carica } \\
\text { papaya) }\end{array}$ & SHR & in vivo & $\begin{array}{l}100 \mathrm{mg} / \mathrm{kg} \text { (twice a } \\
\text { day) }\end{array}$ & $\begin{array}{l}\mathrm{BP} \downarrow \text {, angiotensin converting enzyme(ACE) activity } \downarrow \text {, cardiac } \\
\text { hypertrophy } \downarrow \text {, improve baroreflex sensitivity }\end{array}$ \\
\hline $\begin{array}{l}\text { sour cherry seed } \\
\text { kernel extract }\end{array}$ & $\begin{array}{l}\text { hearts from Sprague- } \\
\text { Dawley rats }\end{array}$ & in vitro & $30 \mathrm{mg} / \mathrm{kg} /$ day & $\begin{array}{l}\text { post ischemic cardiac functions } \uparrow \text {, infarct size } \downarrow \text {, heme oxygenase- I } \\
\text { (HO-I) } \uparrow, \mathrm{Bcl}-2 \uparrow\end{array}$ \\
\hline
\end{tabular}


Table Continued....

\begin{tabular}{|c|c|c|c|c|}
\hline Fruit & Subject & $\begin{array}{l}\text { Study } \\
\text { Type }\end{array}$ & Dose & Main Effects \\
\hline $\begin{array}{l}\text { total flavonoids } \\
\text { of Guangzao } \\
\text { (Choerospondias } \\
\text { axillaris) }\end{array}$ & $\begin{array}{l}\text { I/R male Sprague- } \\
\text { Dawley rats }\end{array}$ & in vivo & $\begin{array}{l}75,150 \text { and } 300 \mathrm{mg} / \\
\mathrm{kg} / \text { day }\end{array}$ & $\begin{array}{l}\text { cardiac function } \uparrow \text {, heart pathologic lesion } \downarrow, \text { CAT } \uparrow, \text { GSH-Px } \uparrow \text {, } \\
\text { SOD } \uparrow, \text { MDA } \downarrow, T \text { NUEL-positive nuclear staining } \downarrow, \mathrm{Bcl}-2 \text {-associated } \\
\text { X protein (Bax) } \downarrow \text {, caspase- } 3 \downarrow, \text { Bcl- } 2 \uparrow, \text { p38 MAPK activity } \downarrow, \text { JNK } \\
\text { activity } \downarrow\end{array}$ \\
\hline $\begin{array}{l}\text { hydroalcoholic } \\
\text { extract of acai } \\
\text { (Euterpe oleracea } \\
\text { Mart.) seeds }\end{array}$ & $\begin{array}{l}\text { male Wistar rats } \\
\text { subjected to } \\
\text { myocardial infarction }\end{array}$ & in vivo & $100 \mathrm{mg} / \mathrm{kg} /$ day & $\begin{array}{l}\text { prevent the development of exercise intolerance, cardiac } \\
\text { hypertrophy, fibrosis, and dysfunction }\end{array}$ \\
\hline Acai pulp & $\begin{array}{l}\text { female Fischer rat } \\
\text { of dietaryinduced } \\
\text { hypercholesterol } \\
\text { emia }\end{array}$ & in vivo & $2 \%(w / w)$ & $\begin{array}{l}\text { TC } \downarrow \text {, LDL- } C \downarrow \text {, atherogenic index } \downarrow, H D L-C \uparrow \text {, cholesterol excretion } \\
\text { in feces } \uparrow \text {, expression of the LDL-R, ABCG5, and ABCG8 genes } \uparrow\end{array}$ \\
\hline $\begin{array}{l}\text { Unrefined black } \\
\text { raspberry seed oils }\end{array}$ & $\begin{array}{l}\text { male Syrian hamsters } \\
\text { fed high-cholesterol } \\
(0.12 \%) \text {, high-fat }(9 \%) \\
\text { diets }\end{array}$ & in vivo & NA & plasma and liver TG $\downarrow$, hypertriglyceridemia $\downarrow$ \\
\hline $\begin{array}{l}\text { Polyphenols from } \\
\text { sea buckthorn } \\
\text { berry }\end{array}$ & $\begin{array}{l}\text { rats with } \\
\text { hyperlipidemia }\end{array}$ & in vivo & $7-28 \mathrm{mg} / \mathrm{kg}$ & $\begin{array}{l}\text { serum lipids } \downarrow \text {,TNF- } \alpha \downarrow \text {, IL- } 6 \downarrow \text {, antioxidant enzymes activity } \uparrow \text {, eNOS, } \\
\text { ICAM-I, and LOX-I mRNA expression and proteins in aortas } \downarrow\end{array}$ \\
\hline $\begin{array}{l}\text { Jujube (Zizyphus } \\
\text { jujuba) fructus and } \\
\text { semen extract }\end{array}$ & human macrophages & in vitro & NA & $\begin{array}{l}\text { the foam cell formation induced by acetylated LDL } \downarrow \text {, prevent } \\
\text { atherosclerosis }\end{array}$ \\
\hline $\begin{array}{l}\text { Yellow passion fruit } \\
\text { pulp }\end{array}$ & SHR & in vivo & 5,6 or $8 \mathrm{~g} / \mathrm{kg} /$ day & SBP $\downarrow, \mathrm{GSH} \uparrow$, thiobarbituric acid-reactive substances (TBARS) $\downarrow$ \\
\hline $\begin{array}{l}\text { Proanthocyanidins } \\
\text { in boysenberry } \\
\text { seed extract }\end{array}$ & $\begin{array}{l}\text { SHR } \\
\text { rat aorta rings }\end{array}$ & $\begin{array}{l}\text { in vivo } \\
\text { in vitro }\end{array}$ & 100 and $200 \mathrm{mg} / \mathrm{kg}$ & $\begin{array}{l}\mathrm{SBP} \downarrow \\
\text { vasorelaxant activity } \uparrow\end{array}$ \\
\hline $\begin{array}{l}\text { Methanolic extract } \\
\text { of date palm } \\
\text { (Phoenix dactylifera } \\
\text { L.) }\end{array}$ & $\begin{array}{l}\text { cerebral ischemia } \\
\text { rats }\end{array}$ & in vivo & $100,300 \mathrm{mg} / \mathrm{kg}$ & $\begin{array}{l}\text { SOD } \uparrow, C A T \uparrow, G S H \uparrow \text {, glutathione reductase } \uparrow \text {, lipid peroxidation } \downarrow \text {, } \\
\text { oxidative stress } \downarrow \text {, neuronal damage } \downarrow\end{array}$ \\
\hline $\begin{array}{l}\text { Black chokeberry } \\
\text { (Aronia } \\
\text { melanocarpa) } \\
\text { extract }\end{array}$ & $\begin{array}{l}\text { bovine coronary } \\
\text { artery endothelial } \\
\text { cells }\end{array}$ & in vitro & $0.1 \mathrm{~g} / \mathrm{mL}$ & NO $\uparrow$, eNOS phosphorylation $\uparrow$ \\
\hline $\begin{array}{l}\text { Saskatoon berry } \\
\text { powder }\end{array}$ & $\begin{array}{l}\text { leptin receptor- } \\
\text { deficient diabetic } \\
\text { mice }\end{array}$ & in vivo & $5 \%(w / w)$ & $\begin{array}{l}\text { monocyte adhesion to aorta } \downarrow \text {, inflammatory, fibrinolytic or stress } \\
\text { regulators in aorta or heart apex } \downarrow\end{array}$ \\
\hline $\begin{array}{l}\text { Saskatoon berry } \\
\text { powder }\end{array}$ & $\begin{array}{l}\text { leptin receptor- } \\
\text { deficient diabetic } \\
\text { mice } \\
\text { glycated LDLtreated } \\
\text { HUVECs }\end{array}$ & in vitro & $5 \%(w / w)$ & $\begin{array}{l}\text { endoplasmic reticulum stress (ERS) } \downarrow \text {, unfolded protein response } \\
\text { (UPR) } \downarrow\end{array}$ \\
\hline $\begin{array}{l}\text { I9 fruits widely } \\
\text { consumed in } \\
\text { central Chile }\end{array}$ & NA & in vitro & $\mathrm{l} \mathrm{mg} / \mathrm{mL}$ & $\begin{array}{l}\text { anticoagulant activities: grape, raspberry fibrinolytic activity: } \\
\text { raspberry }\end{array}$ \\
\hline
\end{tabular}


Table Continued...

\begin{tabular}{|c|c|c|c|c|}
\hline Fruit & Subject & $\begin{array}{l}\text { Study } \\
\text { Type }\end{array}$ & Dose & Main Effects \\
\hline $\begin{array}{l}\text { Peach (Prunus } \\
\text { persica) pulp } \\
\text { ethylacetate } \\
\text { extract }\end{array}$ & $\begin{array}{l}\text { cultured vascular } \\
\text { smooth muscle cells } \\
\text { (VSMCs) }\end{array}$ & in vitro & 50,100 , or $200 \mu \mathrm{g} / \mathrm{mL}$ & $\begin{array}{l}\text { Angiotensin II (Ang II) induced intracellular } \mathrm{Ca}^{2+} \text { elevation } \downarrow \text {, } \\
\text { generation of ROS } \downarrow\end{array}$ \\
\hline $\begin{array}{l}\text { Methanolic extract } \\
\text { of Lingonberry } \\
\text { (Vaccinium vitisidaea } \\
\text { L.) }\end{array}$ & $\begin{array}{l}\text { H9c2 rat myoblasts } \\
\text { simulated IR }\end{array}$ & in vitro & 5 and $10 \mu \mathrm{M}$ & $\begin{array}{l}\text { apoptosis } \downarrow \text {, markers of nuclei condensation, caspase- } 3 \text { activation, } \\
\text { and MAPK signaling } \downarrow\end{array}$ \\
\hline $\begin{array}{l}\text { Blueberry } \\
\text { anthocyanin } \\
\text { fraction (BBA), } \\
\text { blackberry } \\
\text { anthocyanin } \\
\text { fraction (BKA), } \\
\text { and blackcurrant } \\
\text { anthocyanin } \\
\text { fraction (BCA) }\end{array}$ & $\begin{array}{l}\text { RAW } 264.7 \\
\text { macrophages } \\
\text { treated by LPS bone } \\
\text { marrowderived } \\
\text { macrophages from } \\
\mathrm{Nrf2}^{+/+} \text {mice treated } \\
\text { by LPS }\end{array}$ & in vitro & $0-20 \mu \mathrm{g} / \mathrm{mL}$ & $\begin{array}{l}\text { IL-I } \beta \text { mRNA levels } \downarrow \text {, NF- } \kappa B \text { p65 translocation to the nucleus } \downarrow \\
\text { cellular ROS levels } \downarrow \text {, IL- I } \beta \text { mRNA levels } \downarrow\end{array}$ \\
\hline $\begin{array}{l}\text { Pomegranate juice, } \\
\text { together with } \\
\text { date fruit and date } \\
\text { seeds extract }\end{array}$ & apoE $^{-l-}$ mice & in vivo & $\begin{array}{l}0.5 \mu \mathrm{M} \text { gallic acid } \\
\text { equivalents (GAE)/day }\end{array}$ & $\begin{array}{l}\text { TC } \downarrow, \text { TG } \downarrow \text {, PONI activity } \uparrow \text {, mouse peritoneal macrophage (MPM) } \\
\text { oxidative stress } \downarrow \text {, MPM cholesterol content } \downarrow \text {, and MPM LDL } \\
\text { uptake } \downarrow \text {, aortas lipid peroxide content } \downarrow \text {, aortas PON lactonase } \\
\text { activity } \uparrow\end{array}$ \\
\hline
\end{tabular}

\section{Herbs as sources of cardio-protective EMNs}

A few herbs and supplements may help in battling atherosclerosis, the hidden reason for most heart illness. Truth be told, certain herbs can impact pulse, triglycerides, cholesterol levels and irritation, which are all hazard factors for heart malady. They're likewise high in dietary nitrates, which have been appeared to lessen pulse, decline blood vessel firmness and enhance the capacity of cells covering the veins. ${ }^{53}$

\section{Carotenoids}

Carotenoids are a class of normal, fat-dissolvable shades found chiefly in plants. They have potential cancer prevention agent organic properties in view of their compound structure and collaboration with natural films. Carotenoids are generally across the board in the vegetable kingdom and are found in high focuses in algae and microorganisms. People and different animals can't orchestrate them, so they are important in their eating routine. ${ }^{61}$ The scope of uses of microalgae as wellspring of carotenoids isn't constrained to astaxanthin-based items yet in addition to the production of supplements with naturally dynamic carotenoids like $\beta$-carotene, lutein and zeaxanthin with huge commitments to human wellbeing that enter in the sustenance and supplement advertise, which is relied upon to achieve $220 \$$ billion all around in 2022.

Astaxanthin: Astaxanthin (3,3'-dihydroxy- $\beta, \beta^{\prime}$-carotene-4,4'dione), a red carotenoid shade delegated a xanthophyll, is known to have an amazing cell reinforcement capacity. The most astounding known dimension of astaxanthin in nature is in the chlorophyte alga Haematococcus pluvialis. ${ }^{62}$ Astaxanthin utilized in healthful supplements is typically a blend of configurational isomers created by Haematococcus pluvialis, a unicellular microalgae. Astaxanthin controlled orally for five-weeks in stroke inclined SHR additionally brought about a huge BP decrease. Oral astaxanthin likewise improved nitric oxide prompted vascular unwinding in the rodent aortas. ${ }^{63}$ Astaxanthin limits work out prompted skeletal and cardiac muscle harm in mice. ${ }^{64}$ Astaxanthin impacts on pulse in unexpectedly hypertensive rodents (SHR), normotensive Wistar Kyoto rodents (NWKR) and stroke inclined immediately hypertensive rodents (SPSHR) were accounted for. Human umbilical vien endothelial cells and platelets treated with the astaxanthin demonstrated expanded nitric oxide levels and lessening in peroxynitrite levels (Figure 4). ${ }^{65}$

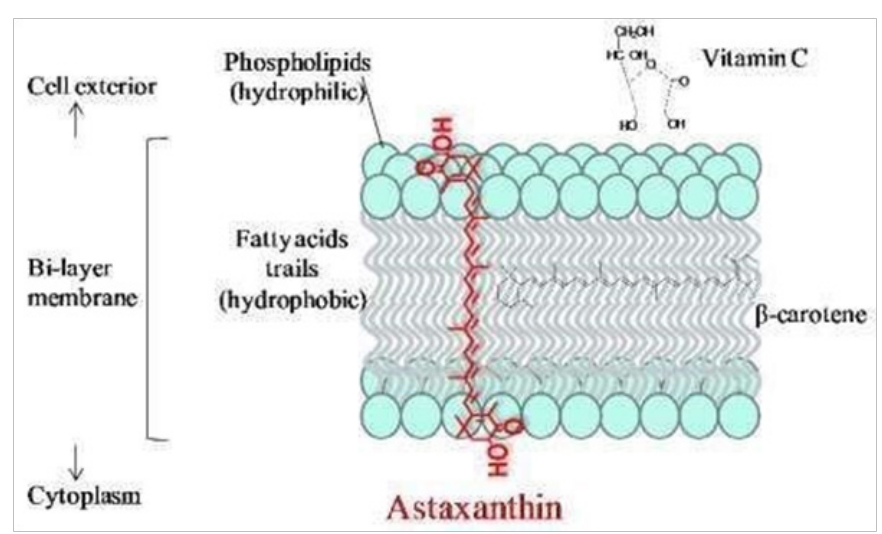

Figure 4 Transmembrane orientation of astaxanthin.

The polar end groups overlap the polar boundary zones of the membrane, while the nonpolar middle fits the membrane's nonpolar interior. The dashed red line speculatively indicates "lightning-rod" conduction of electrons along the astaxanthin molecule, possibly to vitamin $\mathrm{C}$ or other antioxidants located outside the membrane.

Fucoxanthin: Fucoxanthin is an orange carotenoid present in consumable darker ocean growth, for example, Undaria pinnatifida (Wakame), Hijikia fusiformis (Hijiki), Laminaria japonica (MaKombu), and Sargassum fulvellum. ${ }^{61}$ Fucoxanthin, a carotenoid compound, is found in the chloroplasts of darker ocean growth. This phytochemical has very calming and cell reinforcement properties. ${ }^{66}$ Fucoidan and fucoxanthin in mix can possibly lessen cardiac hypertrophy, cardiac fibrosis, ROS level, and abbreviated 
QT interim in maturing mice subjects. There were additionally critical enhancements in cardiac morphology and solid capacity after the maturing mice were bolstered with fucoidan alone or fucoidan supplemented with fucoxanthin. ${ }^{67}$ In addition, fucoxanthin additionally indicated antiobesity, antidiabetes, mitigating, anticancer, and hepatoprotective exercises just as cerebrovascular defensive impacts. Fucoxanthin can enhance the lipid profile and keep the harm in cardiovascular system by advancing the extent of DHA in the liver. ${ }^{68}$ Laminaria japonica and Undaria pinnatifida are among the most well known nourishment elements of Japanese food. Fucoxanthin, and its metabolite fucoxanthinol, lessens inflammatory changes in the connection among adipocytes and macrophages. These outcomes recommend that Fucoxanthin contained in eatable algae is helpful as a sustenance element for controlling corpulence related insulin opposition and for anticipating metabolic disorder (Figure 5)

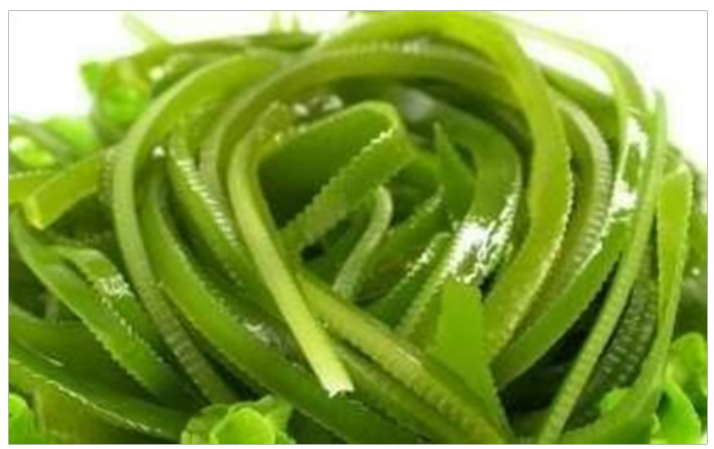

(Figure 6). ${ }^{69}$

\section{Figure 5 Laminaria japonica (Ma-Kombu).}

Laminaria japonica has frequently been used as a food supplement and drug in traditional oriental medicine.Among the major active constituents responsible for the bioactivities of L. japonica, fucoxanthin (FX) has been considered as a potential antioxidant. A low molecular weight fucoidan (DFPS), obtained from the brown seaweed Laminaria japonica, was separated into three fractions by anion-exchange column chromatography. Available data presented the content of sulfate group, the molar ratio of sulfate/fucose and sulfate/total sugar, and the molecular weight played an important role on antioxidant and anticoagulant activity.

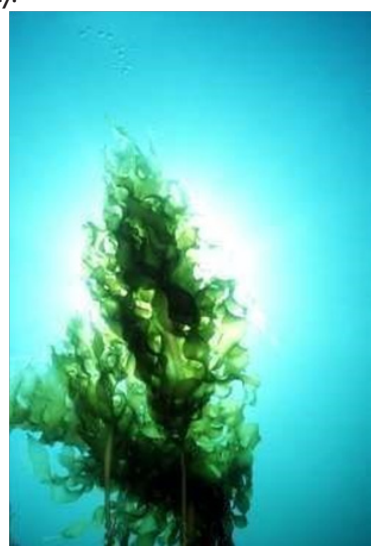

Figure 6 Undaria pinnatifida (Wakame).

Brown algae and its carotenoids have been shown to have a positive influence on obesity and its comorbidities. Additionally, the treatments, ameliorated adipose tissue accumulation, insulin resistance, blood pressure, cholesterol and triglycerides concentration in serum, and reduced lipogenesis and inflammation by downregulating acetyl-CoA carboxylase (ACC) gene expression, increasing serum concentration and expression of adiponectin as well as downregulating IL-6 expression.
Lycopene: Lycopene is the shade in charge of the red shading in a few leafy foods, which can be found in high focus in tomato items, red grapefruits, and watermelons. It is an unsaturated carotenoid, bringing about effective cell reinforcement, and utilization can anticipate both maturing and CVD. ${ }^{61}$ Generally speaking dietary lycopene admission and high-serum grouping of lycopene, altogether diminished the danger of major cardiovascular occasions. ${ }^{70}$ Lutein and lycopene supplementation fundamentally expanded the serum convergence of lutein and lycopene with a lessening in carotid vein CAIMT. ${ }^{71}$ Advantages of lycopene ought to be particularly considered in patients with high cardiovascular hazard, statin prejudice, marginal hypertension, headache medicine opposition, hyperactive platelets, vascular inflammatory infections, metabolic disorder and coronary heart malady, and its incorporation in blend treatments for the referenced issue, ought to be drawn nearer. ${ }^{72}$ Cholesterol decrease, hindrance of oxidation forms, balance of inflammatory markers, upgraded intercellular correspondence, restraint of tumourigenesis and enlistment of apoptosis, digestion to retinoids and antiangiogenic impacts was accounted for in another investigation. ${ }^{73}$ Nonetheless, the conceivable opposite affiliations noted for more elevated amounts of tomato-based items, especially tomato sauce and pizza, with CVD propose that dietary lycopene or different phytochemicals expended as oil-based tomato items give cardiovascular advantages (Figure 7). ${ }^{74}$

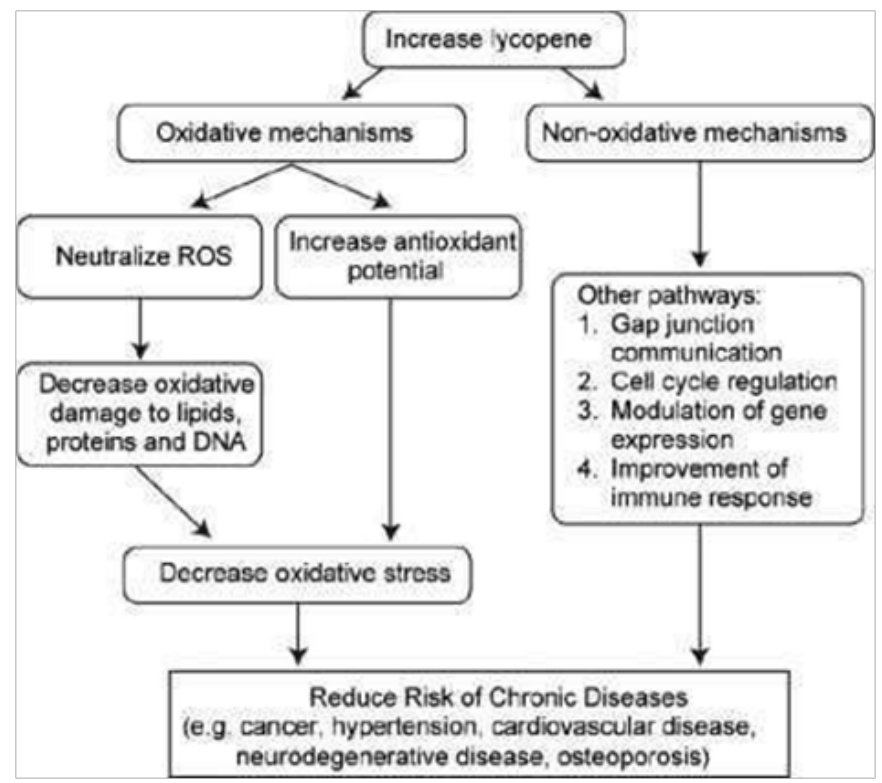

Figure 7 General mechanisms of action of lycopene.

The proposed mechanisms of action of lycopene (oxidative and nonoxidative) that decreases the risk of oxidative stress-mediated diseases. Lycopene most likely acts via the oxidative mechanism of action to prevent oxidative stress. Lycopene treatment has been shown to cause a $37 \%$ suppression of cellular cholesterol synthesis in J-774A.I macrophage cell line, and augment the activity of macrophage LDL receptors. Oxidized LDLs are highly atherogenic as they stimulate cholesterol accumulation and foam cell formation, initiating the fatty streaks of atherosclerosis. LDL susceptibility to oxidative modifications is decrease by an acyl analog of platelet-activating (PAF), acyl-PAF, which experts its beneficial role during the initiation and progression of atherosclerosis. Purified lycopene in association with $\alpha$ - tocopherol or tomato lipophilic extracts has been shown to enhance acyl-PAF biosynthesis in endothelial cells during oxidative stress. ROS: reactive oxygen species.

Lutein: It is a pigment (xanthophyll) and a dietary oxygenated carotenoid comprising of 40-carbon hydroxylated compounds found in the human retina in high fixation. It is an isomer of the 
carotenoid zeaxanthin, with indistinguishable compound equations. ${ }^{61}$ it can simply be gotten from yellow corn, egg yolk, squeezed orange, honeydew melon, and different organic products, however particularly happening in dim green vegetables, for example, turnip greens, kale, parsley, spinach, and broccoli. ${ }^{75}$ Lutein applies powerful cell reinforcement and calming impacts in aortic tissue that may secure against advancement of atherosclerosis in guinea pigs. ${ }^{76}$ utilization of lutein expands plasma lutein fixations, and that this expansion is related with increments in movement and decreases in time spent occupied with inactive exercises..$^{77}$ Lutein may go about as a chemopreventive specialist against atherosclerosis, including oxidative pressure and lipid digestion upgrades. Diminished mRNA and protein articulation dimensions of hepatic peroxisome proliferator-enacted receptor- $\alpha$, carnitine palmitoyl-transferase $1 \mathrm{~A}$, acyl CoA oxidase 1 , low thickness lipoprotein receptors and forager receptor class B type I saw in mice with atherosclerosis were extraordinarily upgraded after treatment with lutein. ${ }^{78}$

Zeaxanthin: Like lutein, zeaxanthin is an oxygenated non-ace nutrient A carotenoid that comprises of a 40-carbon hydroxylated compound and they both are found from same dietary sources ${ }^{79}$ Higher dietary and serum carotenoid levels are related with lower carotid intima-media thickness in moderately aged and older individuals. ${ }^{80}$ More elevated amounts of plasma oxygenated carotenoids (lutein, zeaxanthin, betacryptoxanthin) and alpha-carotene might be defensive against early atherosclerosis. ${ }^{81}$ Specialists found a connection between the dimension of lutein and zeaxanthin in the fat tissue and diet and the hazard for heart assault. ${ }^{82}$

$\boldsymbol{\beta}$-cryptoxanthin: $\beta$-cryptoxanthin is a xanthophylls and one of the lesser-known carotenoids, whose best sustenance sources are oranges, peach, tangerines, red peppers and tropical organic products, for example, papaya and pumpkin. It likewise has ace nutrient an action and appears to have defensive wellbeing activity. The centralizations of $\beta$-cryptoxanthin in most mammalian tissues commonly are low contrasted and those of other dietary cancer prevention agents, for example, nutrients $\mathrm{E}$ and $\mathrm{C} .^{83}$ Intense $\beta$-cryptoxanthin treatment shows more prominent cardioprotective viability against $I / R$ damage than astaxanthin and nutrient $\mathrm{E}$ by diminishing infarct sizes and lessening apoptosis, oxidative pressure, and mitochondrial brokenness in mice. ${ }^{84}$ Serum $\beta$-cryptoxanthin and lutein in addition to zeaxanthin were conversely identified with the degree of atherosclerosis. ${ }^{85}$ $\beta$-cryptoxanthin has hostile to corpulence and antioxidative impacts in $C$. elegans, it is enticing to conjecture that $\beta$-cryptoxanthin may have a defensive impact against advancement of the metabolic disorder. The metabolic disorder is a mind boggling jumble bunching stoutness, diabetes mellitus, and atherosclerotic cardiovascular maladies. $\beta$-cryptoxanthin fixations in serum are contrarily identified with files of oxidative DNA harm and lipid peroxidation. ${ }^{86} \mathrm{~A}$ converse relationship of baPWV with $\beta$-carotene and $\beta$-cryptoxanthin was watched freely of the glycemic state. ${ }^{87}$

Beta-carotene: Beta-carotene is a standout amongst the most generally examined carotenoids for the two its genius nutrient $\mathrm{A}$ movement and its wealth in foods grown from the ground, for example, carrot, orange, kale, spinach, turnip greens, apricot, and tomato. ${ }^{61}$ Low serum carotenoid levels may reflect either expanded lipoprotein thickness or the nearness of irritation, the two factors developing as imperative novel hazard factors for coronary heart infection. ${ }^{88}$ Notwithstanding its capacity to influence hazard and pathogenesis of cancer, beta-carotene has been considered for use in the executives of heart ailment dependent on its free radical searching limits, with the preventative note that it might likewise go about as a tissue-harming prooxidant-contingent upon the physiologic environment. ${ }^{89,90}$ Clear cardioprotective impact of beta-carotene at one chose measurements and moderation or end of that security at a higher portion is theoretical dependent on the results. ${ }^{91}$ One-month tobacco-smoke introduction instigates practical and morphological cardiac adjustments and $\mathrm{BC}$ supplementation weakens this ventricular redesigning process..$^{92}$ More data is expected to determine the relationship between the admission of single supplements, for example, carotenoids, and the danger of CVD. As of now, the utilization of carotenoids in pharmaceutical structures for the treatment or aversion of heart infections can't be prescribed. ${ }^{93}$

\section{Homocysteine level maintenance}

Homocysteine levels increment in the body when the digestion to cysteine of methionine to cysteine is impeded. This might be because of dietary inadequacies in nutrient B6, nutrient B12, and folic acid. ${ }^{94}$ Lifted homocysteine level has been appeared to be related with the advancement of atherosclerotic heart illness, stroke, and myocardial ischemia. An ascent in serum creatinine likewise prompts an ascent in fasting all out homocysteine. The real course of homocysteine freedom from plasma is the kidney, and the ascent is because of inadequate digestion of homocysteine by the kidney. ${ }^{95} \mathrm{~A} 25 \%$ decrease in homocysteine levels was related with a $11 \%$ lower ischemic heart ailment chance and a $19 \%$ lower stroke chance. ${ }^{96}$ Trimethylglycine, Folate, Vitamin B12, Vitamin B6, Taurine, Creatine, Choline, NAcetylCysteine, Omega-3 Fatty Acids hold homocysteine levels under tight restraints (Figure 8). ${ }^{97,98}$

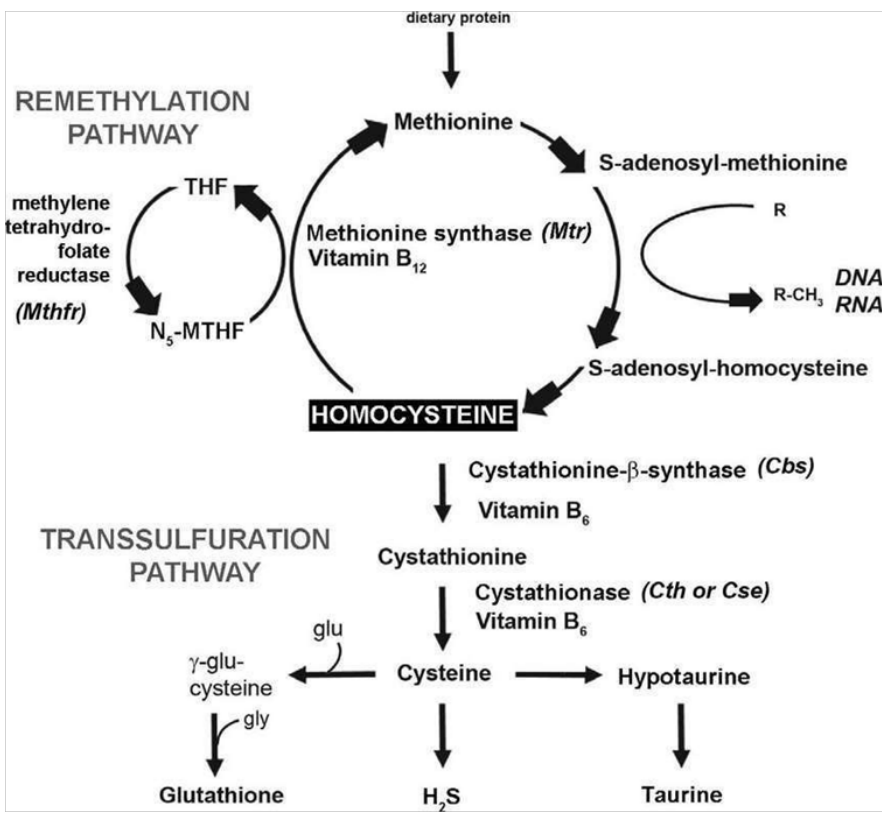

Figure 8 Pathways for the metabolism of homocysteine

Normal trans-sulfuration requires cystathionine $\beta$ synthase with vitamin B6 as cofactor. Re-methylation requires 5, 10-methylenetetrahydrofolate reductase and methionine synthase.The latter requires folate as co-substrate and vitamin BI2 (cobalamin) as cofactor. An alternative remethylation pathway also exists using the cobalamin independent betaine-homocysteine methyltransferase. For some years, attention focused on the role of heterozygosity for homocystinuria 6 as a possible cause of the high homocysteine concentrations that is seen in up to $30-40 \%$ of patients with coronary artery disease.

\section{Flavonoids}

An extensive assemblage of proof backings that the dietary admission of polyphenols-especially of flavonoids and the particular class of flavonoids named flavanols-may almost certainly apply some 
gainful vascular impacts and lessen the hazard for cardiovascular horribleness and mortality. ${ }^{99}$ Various flavonoids of dietary criticalness have been appeared to give valuable effect on parameters related with atherosclerosis, including lipoprotein oxidation, blood platelet conglomeration and cardiovascular reactivity. ${ }^{100} \mathrm{In}$ an investigation of around 5,000 subjects, the admission of dietary flavonoids and tea was contrarily connected with myocardial dead tissue. ${ }^{101}$ Flavonoids' defensive impacts against ischemic heart illness depends on a few clinical examinations that decidedly connect flavonoid admission to a decreased occurrence of the infection. Expending flavonoids like catechin, which is available in plant seeds and teas, brought about a $20 \%$ decrease in the frequency of the sickness ${ }^{102}$ (Further subtleties of flavonoids in CVD avoidance is talked about in later area) (Figure 9).

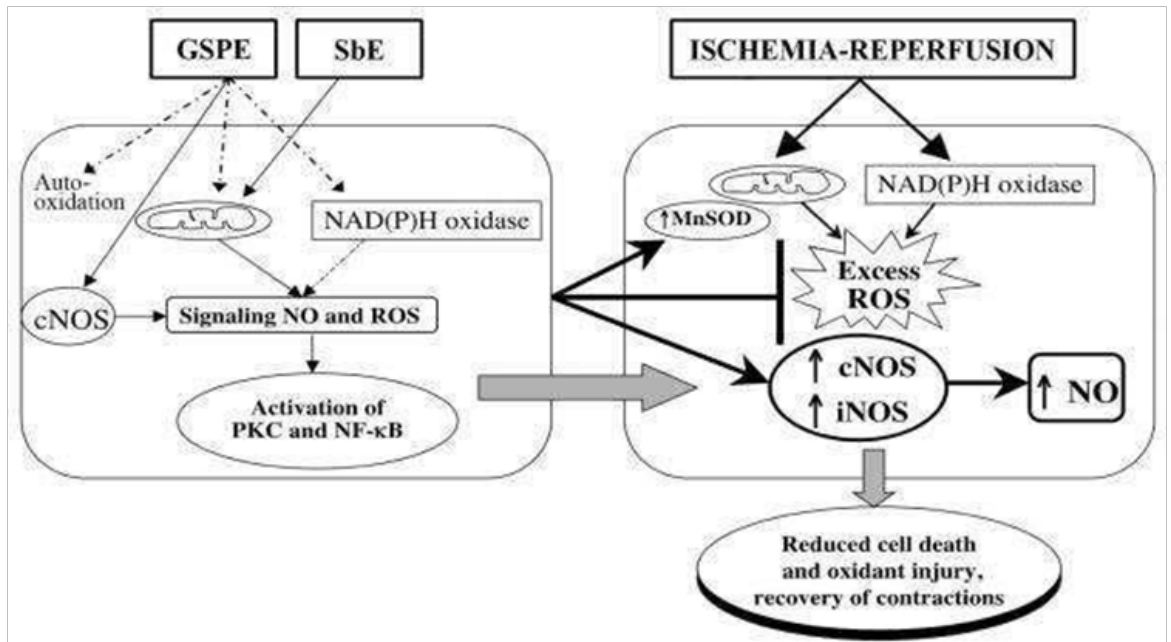

Figure 9 Potential mechanisms of Scutellaria baicalensis extract (SbE) and grape seed proanthocyanidin extract (GSPE) in delayed preconditioning in cardiomyocytes.

NO, nitric oxide; ROS, reactive oxygen species; PKC, protein kinase C; MnSOD, manganese superoxide dismutase; cNOS, constitutive nitric oxide synthase; iNOSinducible NOS. With respect to NO, flavonoids in SbE such as wogonin and baicalein, suppress its release by inhibiting NOS/guanylate cyclase. In normal tissue GSPE stimulates NO release via a purinergic pathway. GSPE has shown a reduced NO release, although only in models of inflammation, which could be an indirect effect of suppression of iNOS upregulating cytokines. Data suggest that cardiomyocytes show a non-toxic ROS response to SbE treatment but NO release with GSPE treatment during the induction phase. It appears that the two extracts may induce two distinct preconditioning mechanisms.

\section{Magnesium and iron}

The role of copper, iron, and other metal components in ischemic heart damage has been settled. ${ }^{103}$ Vegetables (lentils, beans and peas) pumpkin, sesame, hemp and flaxseeds, cashews, pine nuts and different nuts, tomato, potatoes, mushrooms, palm, prune, olives, mulberries, entire, grains (amaranth, oats), coconut milk, dull chocolate, dried thyme are rich wellspring of iron. ${ }^{104,105}$ Brazil nuts, almonds, pecans, cashews, walnuts, pumpkin seeds, flaxseeds, sunflower seeds, sesame seeds, quinoa seeds, cumin seeds, peach apricots, avocado, banana, blackberries, spinach, okra, broccoli, beetroot, swiss chard, green chime peppers, artichokes, buckwheat are rich wellsprings of magnesium. ${ }^{106}$ Iron is a segment of hemoglobin and subsequently assumes a key job in tissue oxygenation. It is additionally a segment of myoglobin, which is an oxygen restricting protein found in skeletal muscle and myocytes, permitting oxygen discharge in hypoxic conditions. ${ }^{107}$ The commonness of iron insufficiency in heart disappointment patients has been accounted for as being up to $50 \%$, even in patients without sickliness. In this way, IV iron ought to be considered in symptomatic HF patients with ID. ${ }^{108}$ Examination of the long haul security of the different intravenous iron supplementation techniques may in any case be justified (Table 5). ${ }^{109}$

Table 5 Summary of studies evaluating the effect of magnesium on cardiovascular-related outcomes in the general population ${ }^{104}$

\begin{tabular}{|c|c|c|c|c|}
\hline Study type & Clinical setting & $\begin{array}{l}\text { No. of } \\
\text { Subjects }\end{array}$ & Outcome & Conclusion \\
\hline $\begin{array}{l}\text { Meta-analysis of } \\
\text { prospective studies }\end{array}$ & General population & $>1,000,000$ & $\begin{array}{l}\text { CVD (coronary heart disease, ischemic } \\
\text { heart disease, stroke) and allcause } \\
\text { mortality }\end{array}$ & $\begin{array}{l}\text { Increasing dietary } \mathrm{Mg} \text { is associated with a } \\
\text { reduced risk of stroke and heart failure, but } \\
\text { not with total CVD, and all-cause mortality. }\end{array}$ \\
\hline Observational & Elderly & 1400 & All-cause and causespecific mortality & $\begin{array}{l}\text { Low plasma Mg levels increase allcause } \\
\text { mortality. }\end{array}$ \\
\hline $\begin{array}{l}\text { Meta-analysis of } \\
\text { prospective studies }\end{array}$ & General population & 532,979 & CVD & $\begin{array}{l}\text { Inverse association between dietary } \mathrm{Mg} \\
\text { intake and CVD risk. }\end{array}$ \\
\hline $\begin{array}{l}\text { Meta-analysis of } \\
\text { prospective studies }\end{array}$ & General population & $313,04 \mid$ & Incidence of CVD, including IHD & $\begin{array}{l}\text { Plasma and dietary } \mathrm{Mg} \text { are inversely } \\
\text { associated with CVD risk. }\end{array}$ \\
\hline Prospective & $\begin{array}{l}\text { Individuals at high } \\
\text { risk of CVD }\end{array}$ & 7216 & CVD and all-cause mortality & $\begin{array}{l}\mathrm{Mg} \text { intake is associated with a lower } \\
\text { mortality risk in this population, but not } \\
\text { with } \mathrm{CV} \text { events. }\end{array}$ \\
\hline Prospective & $\begin{array}{l}\text { Women free of } \\
\text { disease }\end{array}$ & 86,323 & $\mathrm{CHD}$ & $\begin{array}{l}\text { Dietary } \mathrm{Mg} \text { intake was inversely associated } \\
\text { with fatal } \mathrm{CHD} \text {. }\end{array}$ \\
\hline
\end{tabular}




\section{Omega-3 polyunsaturated fatty acids}

Early secondary counteractive action preliminaries of fish and omega-3 polyunsaturated unsaturated fat (PUFA) cases announced useful impacts on CVD results, including all-cause mortality and sudden cardiac passing. ${ }^{110}$ Other than numerous medical advantages, incomprehensibly; extraordinary exercise can result in oxidative harm to cell constituents. Very still, muscle gets roughly $20 \%$ of the absolute blood stream, be that as it may, amid exercise, this can increment to over $80 \%$. N-3 PUFAs appear to be among the most valuable supplements for an immense scope of the populace (untimely babies, old with sarcopenia, competitors, and patients with metabolic and inflammatory maladies). N-3 PUFAs can possibly be an ergogenic help that improves preparing and sport execution requiring little to no effort and little hazard. ${ }^{111}$ Omega3 unsaturated fats like EPA just at a pharmacologic portion diminish fasting TG and meddle with components of atherosclerosis that outcomes in decreased cardiovascular occasions. Extra robotic preliminaries will give further bits of knowledge into their job in decreasing cardiovascular hazard in subjects with all around oversaw LDL-C yet lifted TG levels (Figure $10) .{ }^{112}$

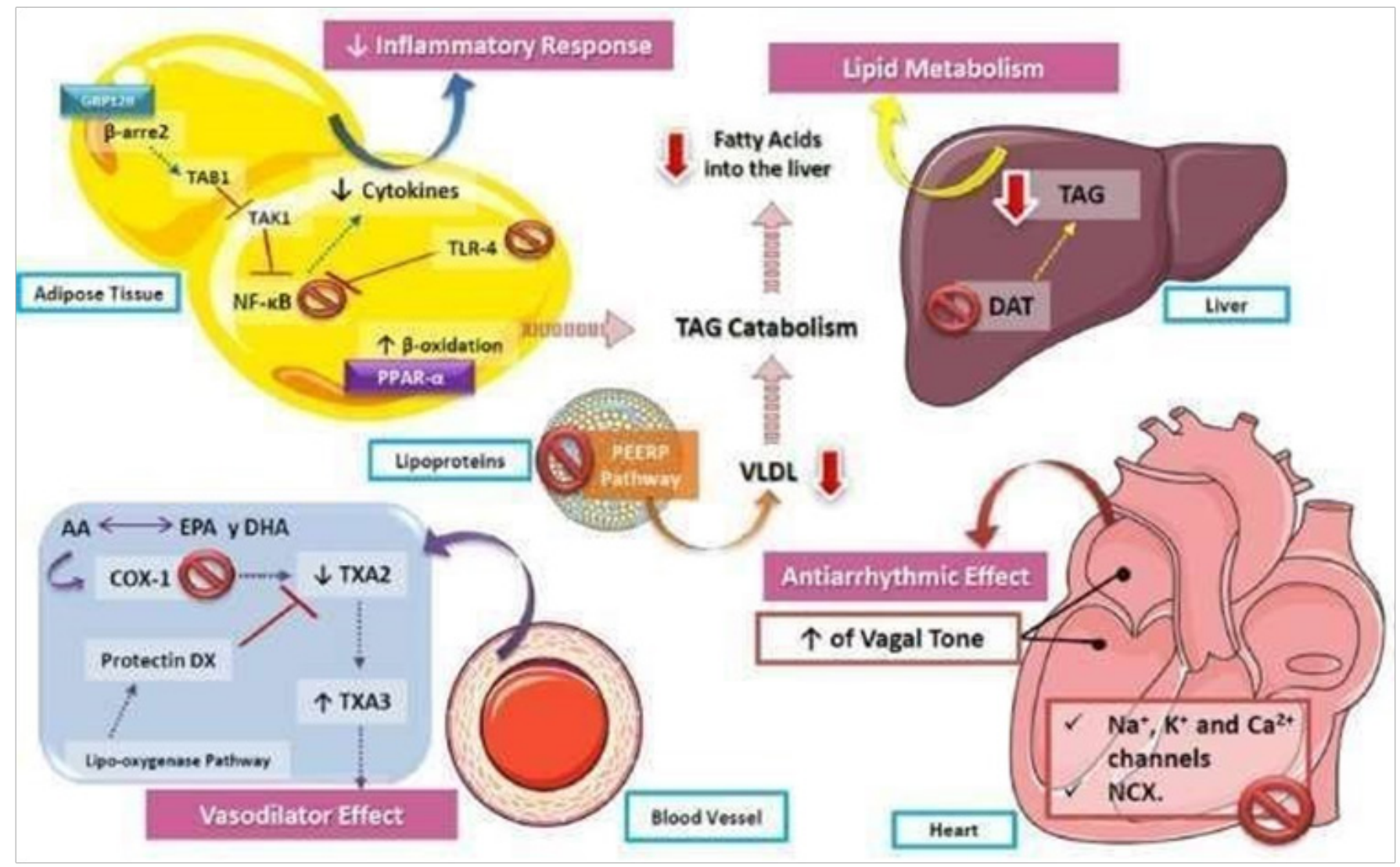

Figure 10 Role of polyunsaturated fatty acids in cardiovascular function

The actions of $n 3$-PUFAs are diverse, including the decrease of the inflammatory response via NF-kB inhibition, as well as an increase of $\beta$-oxidation, causing the catabolism of triaclyglycerides and contributing to the decrease of lipids stored both in the liver and vessel walls. In addition, by increasing the production of TXA3 in vessel walls, PUFAs decrease vascular resistance, reducing blood pressure. On the other hand, one of the most described effects of n3-PUFAs is their action on cardiac arrhythmia, by inhibiting voltage-gated ion channels and exchangers, as well as increasing the vagal tone of the atria and ventricles, which leads to a lower heart rate. PUFA, polyunsaturated fatty acids; TXA2, thromboxane A2; TXA3, thromboxane A3, COX-I: cyclooxygenase I; DAT, I,2 diglyceride acyltransferase;TAG, triacylglycerides; NF-kB, nuclear factor kappa B;TLR-4, toll-like receptor 4;VLDL, very low-density lipoproteins.

\section{Co-enzyme IO (QI0)}

CoQ10 isn't FDA-endorsed to treat any restorative condition in spite of the fact that it is generally accessible over-the-counter as a dietary supplement and prescribed by primary consideration doctors and pros alike. ${ }^{113}$ Q10 can build the creation of key cell reinforcements, for example, superoxide dismutase, a chemical fit for lessening vascular oxidative worry in hypertensive patients. Q10 diminishes dimensions of lipid peroxidation through the decrease of ace oxidative compounds. Q10 can upgrade blood stream and ensure veins by means of the safeguarding of nitric oxide. ${ }^{114}$ There's a solid job of CoQ10 in hypertension, ischemic heart malady, myocardial dead tissue, heart disappointment, viral myocarditis, cardiomyopathies, cardiac poisonous quality, dyslipidemia, corpulence, type 2 diabetes mellitus, metabolic disorder, cardiac methodology and revival. ${ }^{115}$ Q10 has the potential in hypertensive patients to bring down systolic circulatory strain by up to $17 \mathrm{~mm} \mathrm{Hg}$ and diastolic pulse by up to $10 \mathrm{~mm} \mathrm{Hg}$ without noteworthy reactions. ${ }^{116}$ Proof proposes that the CoQ10 supplement might be a valuable apparatus for overseeing patients with heart disappointment. ${ }^{117}$ In spite of positive discoveries, a bigger imminent preliminary is justified to help routine utilization of CoQ10 (Figure 11). ${ }^{118}$ 


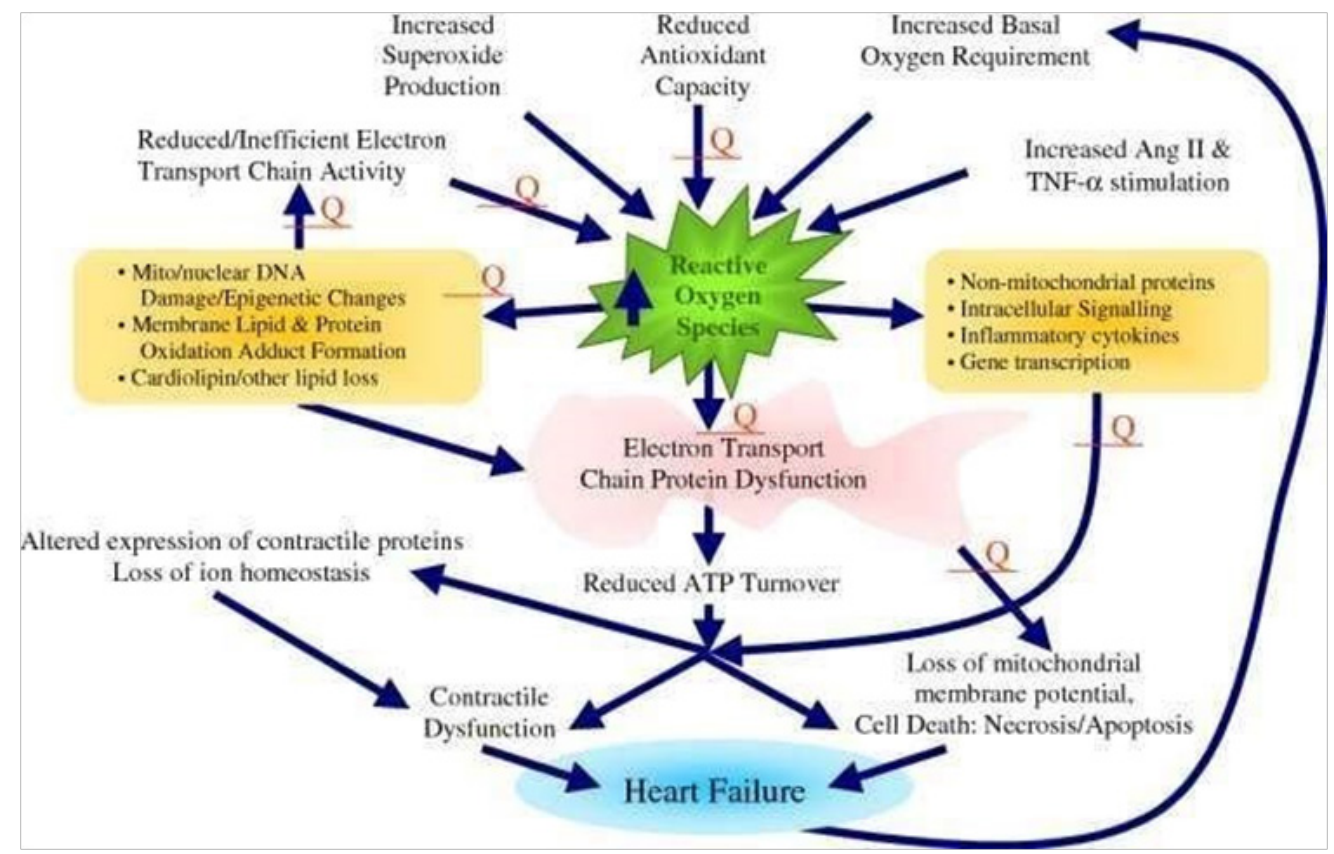

Figure I I QI0 treatment may intervene in the scheme where augmented ROS production contributes to postischemic injury and progression to heart failure.

Reactive oxygen species (ROS) Increased myocardial levels of oxidative stress markers have been demonstrated in animal models of heart failure produced by coronary ligation, pressure overload and rapid cardiac pacing. ROS are key pathophysiological mediators in myocardial remodeling in heart failure. In human heart failure, there is also evidence of increased levels of oxidative stress markers such as malondialdehyde (MDA) in serum, and isoprostanes in urine. Furthermore the levels of these markers correlate with the severity of heart failure.

\section{Indian herbs for the CVD management}

In traditional medicinal systems, hypertension is analyzed by its evident side effects. The traditional mending additionally portrays different side effects, for example, serious migraine, weariness, chest torment; sporadic heart beat among others for a determination of cardiac illnesses. ${ }^{119}$ A recent report demonstrated that $25 \%$ of present day medication and $75 \%$ of new medicines against harmful ailments are acquired from normal plant assets. ${ }^{120}$

\section{Allium sativum (Family: Alliaceae or Liliaceae)}

The protective mechanisms of the beneficial effects of garlic in CVDs may be achieved by suppressing LDL oxidation, increasing HDL, as well as decreasing TC and TG. ${ }^{121}$ While garlic supplementation reduced BP significantly in hypertensive patients, it did not appreciably affect patients with normal BP (Figure 12). ${ }^{122}$

\section{Terminalia arjuna (Family: Combretaceae)}

Bark of T. arjuna contains an extremely abnormal state of flavonoids, to be specific arjunolone, flavones, luteolin, baicaleiin, quercetin, kempferol, and pelargonidin assessed with other medicinal plants especially effect sly affecting cardiovascular sicknesses. ${ }^{123} \mathrm{~T}$. arjuna is broadly utilized for treatment of cardiovascular sicknesses, including heart illnesses and related chest torment, hypertension and elevated cholesterol. Various clinical investigations have likewise detailed its helpful impacts in patients of interminable stable angina, endothelial brokenness, heart disappointment and even ischemic mitral spewing forth. ${ }^{124}$ Its bark decoction is being utilized in the Indian subcontinent for anginal agony, hypertension, congestive heart disappointment, and dyslipidemia, in view of the perceptions of antiquated doctors for quite a long time. ${ }^{125}$ No systematic survey has been directed for Terminalia arjuna in patients of ceaseless stable angina. ${ }^{126}$ It sustains and reinforces the heart muscle and advances cardiac working by directing pulse and cholesterol. ${ }^{127} \mathrm{~T}$. arjuna essentially diminishes TC, LDL and TG levels and expands HDL and decreases atherosclerotic injury in aorta of hypercholesterolemic rabbits. ${ }^{128}$ The adequacy of $\mathrm{T}$. arjuna stem bark as a cardioprotective and intense cancer prevention agent has been adequately shown in various trial and clinical investigations. Be that as it may, persistent research advance on $\mathrm{T}$. arjuna stem bark is especially required in the respect of careful sub-atomic system, tranquilize organization, medicate sedate associations, and toxicological investigations (Figure 13). ${ }^{129}$

\section{Allium cepa (Onion)}

Onion strip extract supplementation for about fourteen days is advantageous as it diminishes the likelihood of creating key hazard factors for cardiovascular ailment by modifying the lipid profiles in sound young ladies. ${ }^{130}$ Garlic oil and onion oil have hostile to stoutness properties that can neutralize the impacts of a HFD on body weight, fat tissue weight, and serum lipid profiles. ${ }^{131}$ HPLC investigation of onion strip separate uncovered that it contains quercetin, one of the real flavonoids, which has hostile to platelet impact (a powerful inhibitor of collagen-invigorated platelet conglomeration in vitro), along these lines, it very well may be a promising and safe methodology for against cardiovascular sicknesses. ${ }^{132}$ Hyperglycemia has been recognized as a noteworthy hazard factor for cardiovascular intricacies connected to T2DM, and in this way known as a compelling restorative focus in the treatment of T2DM. Warmth handled onion concentrate can be a critical wellspring of arginyl-fructose, a noteworthy bioactive Amadori improvement compounds in warmth prepared onion, and phenolic compounds that apply postprandial blood glucosebringing down and cancer prevention agent impacts, separately. ${ }^{133}$ Organization of garlic in addition to lemon juice brought about an 
enhancement in lipid levels, fibrinogen and circulatory strain of patients with hyperlipidemia. ${ }^{134}$ Onion globules (Allium cepa L.) are among the most extravagant wellsprings of dietary flavonoids and add to a substantial degree to the general admission of flavonoids (Figure 14). ${ }^{135-137}$

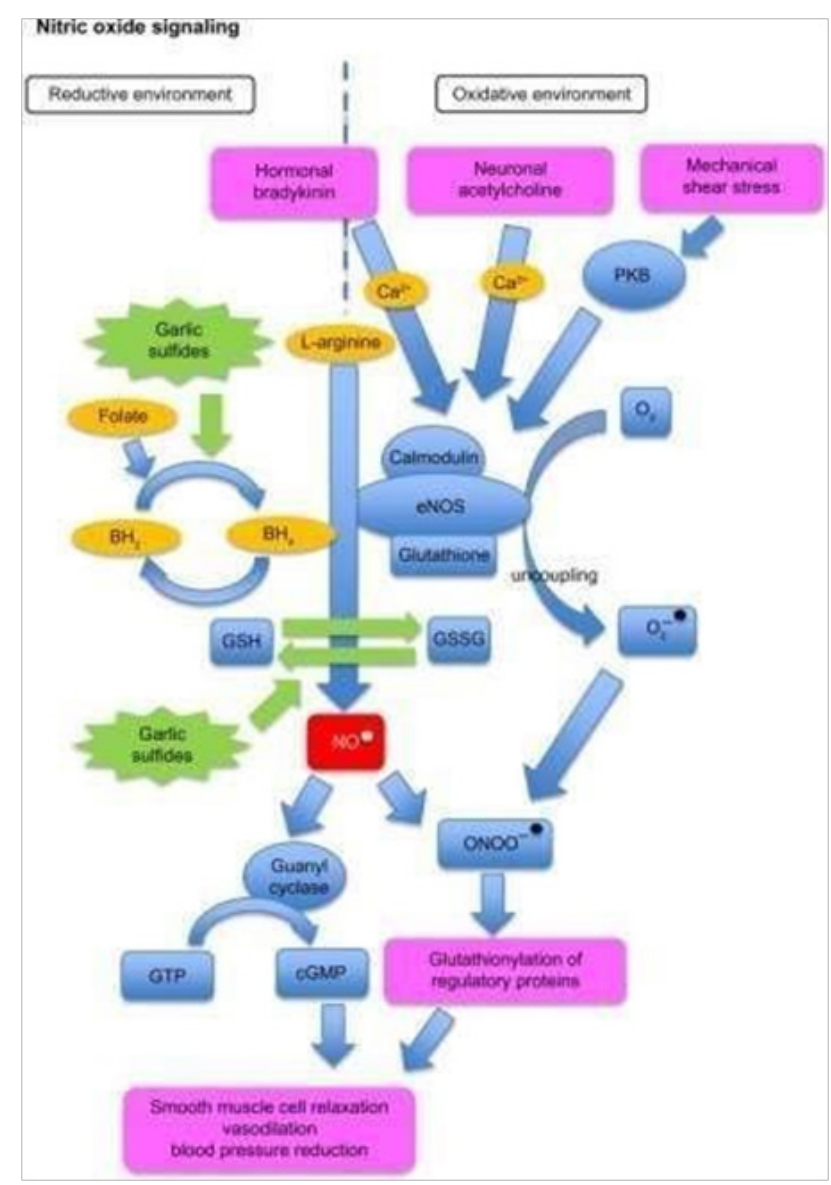

Figure 12 Effect of garlic on blood pressure via the NO pathway.

N.B. Blue rectangles illustrate metabolites, blue circles represent enzymes, orange circles are dietary cofactors, green star shapes are garlic and other organo sulfur containing nutrients, red rectangle represents NO, and purple rectangles denote direct and indirect influence of $\mathrm{NO}$ on vasodilation and blood pressure. $\mathrm{NO}$ pathway: in the presence of $\mathrm{BH}_{4}$, eNOS produces $\mathrm{NO}$, which triggers pathways leading to smooth muscle cell relaxation and vasodilation. eNOS uncoupling leads to the formation of $\mathrm{O}_{2}^{-}$. $\mathrm{NO}$ and $\mathrm{O}_{2}{ }_{2}^{-}$combine to form $\mathrm{OONO}^{-}$, which rapidly reacts with thiols and tyrosine residues of proteins, which in turn, leads to vasodilation and BP reduction independent of cGMP. Garlic and other dietary organosulfides may play a role in the regulation of the NO signaling pathway by creating a more reductive environment and therefore supporting NO production. Abbreviations: $\mathrm{BH} 2$, dihypdrobiopterin; $\mathrm{BH} 4$ tetrahydrobiopterin; $\mathrm{Ca}^{2+}$ calcium ion; cGMP, cyclicguanosyl-monophosphate; GSSG, oxidized glutathione; eNOS, endothelial-nitric-oxide-synthase; GSH, reduced free glutathione; GTP, guanosyl-tri-phosphate; NO, nitric oxide (radical); ONOO, peroxynitrite; $\mathrm{O}_{2}$, oxygen; $\mathrm{O}_{2}^{-}$, superoxide anion radical; PKB, protein kinase-B.

\section{Amla (Emblica officinalis)}

Amla delivered critical hypolipidemic impact alongside a decrease in circulatory strain. Expansion of Amla to the as of now accessible hypolipidemic treatment would offer huge insurance against atherosclerosis and coronary conduit infection, with decrease in the portion and antagonistic impacts of the hypolipidemic operators. ${ }^{138}$ It has useful job on dyslipidemia and cardiac autonomic capacities in rodents treated with high fat eating regimen. ${ }^{139} \mathrm{~A}$ strong hindrance of collagen prompted platelet collection because of oral supplementation of an institutionalized concentrate of Phyllanthus emblica. Furthermore, the concentrate fundamentally repressed hypercholesterolemia and hs-CRP in overweight/Class-1 large grown-ups from the US populace. ${ }^{140}$ In a recent report, Amla remove has appeared potential in decreasing TC and TG levels just as lipid proportions, AIP and apoB/apo An I in dyslipidemic people (apo $\mathrm{B}$, apo An I is a noteworthy apolipoprotein of HDL particles which enables the inversion to transport of cholesterol from fringe tissue to liver, in this manner lessening the danger of creating inflammatory reaction and development of plaques) and in this manner has degree to regard general just as diabetic dyslipidemia. A solitary specialist to lessen cholesterol just as TG is uncommon. Cholesterol decrease is accomplished without corresponding decrease of Co Q10, as opposed to what is seen with statins (Figure 15). ${ }^{141,142}$

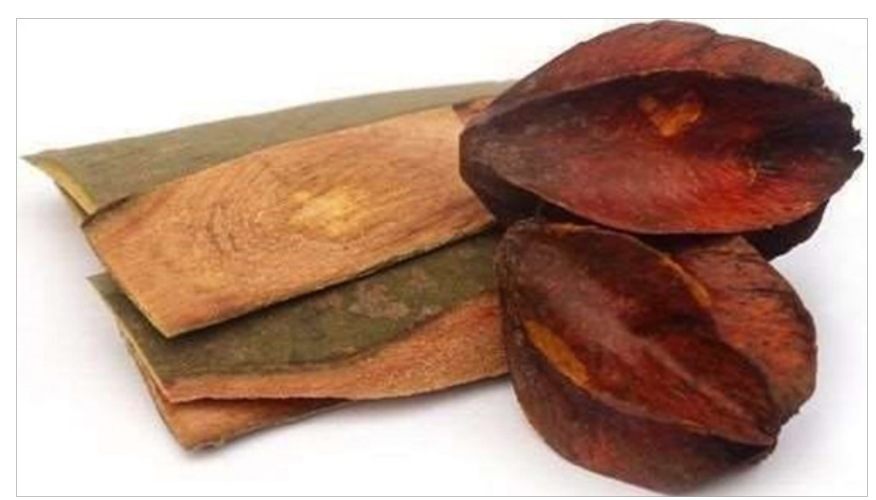

Figure 13 Medicine Grade Terminalia arjuna Bark and Dried Fruits (Source: Indiamart).

\section{Withania somnifera (Ashwagandha)}

Ashwagandha root extricate upgrades the Cardiorespiratory perseverance and enhances QOL in sound athletic grown-ups. ${ }^{143}$ Ashwagandha is appeared in the writing to be an anxiolytic, upper, and antistress adaptogen. Various examinations have affirmed the antistress impact of ashwagandha. ${ }^{144-147}$ It additionally seems to apply a positive effect on the endocrine, cardiopulmonary, and focal sensory systems. ${ }^{148}$ avoided increment in adrenal weight and diminishing in ascorbic acid and Cortisol substance of adrenals amid stress. It seems to incite a condition of non-explicitly expanded obstruction (SNIR) amid stress (Figure 16) (Figure 17). ${ }^{149}$

\section{Nardostachys jatamansi (Valerianaceae)}

Possesses significant anti-stress activity, which might be because of its cancer prevention agent action. ${ }^{150}$ In vitro cell reinforcement action of hydromethanolic extricates $(70 \%)$ of $\mathrm{N}$. jatamansi was considered by estimating the free radical searching movement. Rakatchap Har (Each $500 \mathrm{mg}$ top contains Sarpgandha 150mg, Shankhpushpi 75mg, Jatamansi 75mg, Jahar Mohra Khatai Pishti $75 \mathrm{mg}$, Moti Pishti 75mg, Ras Sindoor 50mg) alongside way of life alteration and psychotherapy is a sheltered and useful solution for the treatment of all evaluations of hypertension in all age bunches with no constraint to its utilization. ${ }^{151}$ Brahmyadi Churna (Brahmi, Shankhapushpi, Jatamansi, Jyotishmati, Vacha, Ashwagandha Churna 1 section each) caused stamped decrease in the dimensions of all out BP. Manifestations, for example, migraine, sleep deprivation, and energy indicated stamped enhancement while very little decrease was 
seen in different side effects, for example, chest agony, exhaustion, and palpitation. SBP diminished impressively than DBP reduction in $\mathrm{BP}$ was watched notably with $\mathrm{P}<0.000 .{ }^{152}$ The fluid concentrates additionally shown defensive impacts against $2 \mathrm{~K} 1 \mathrm{C}$-instigated cardiac hypertrophy in a rodent demonstrate (Figure 18). ${ }^{153}$

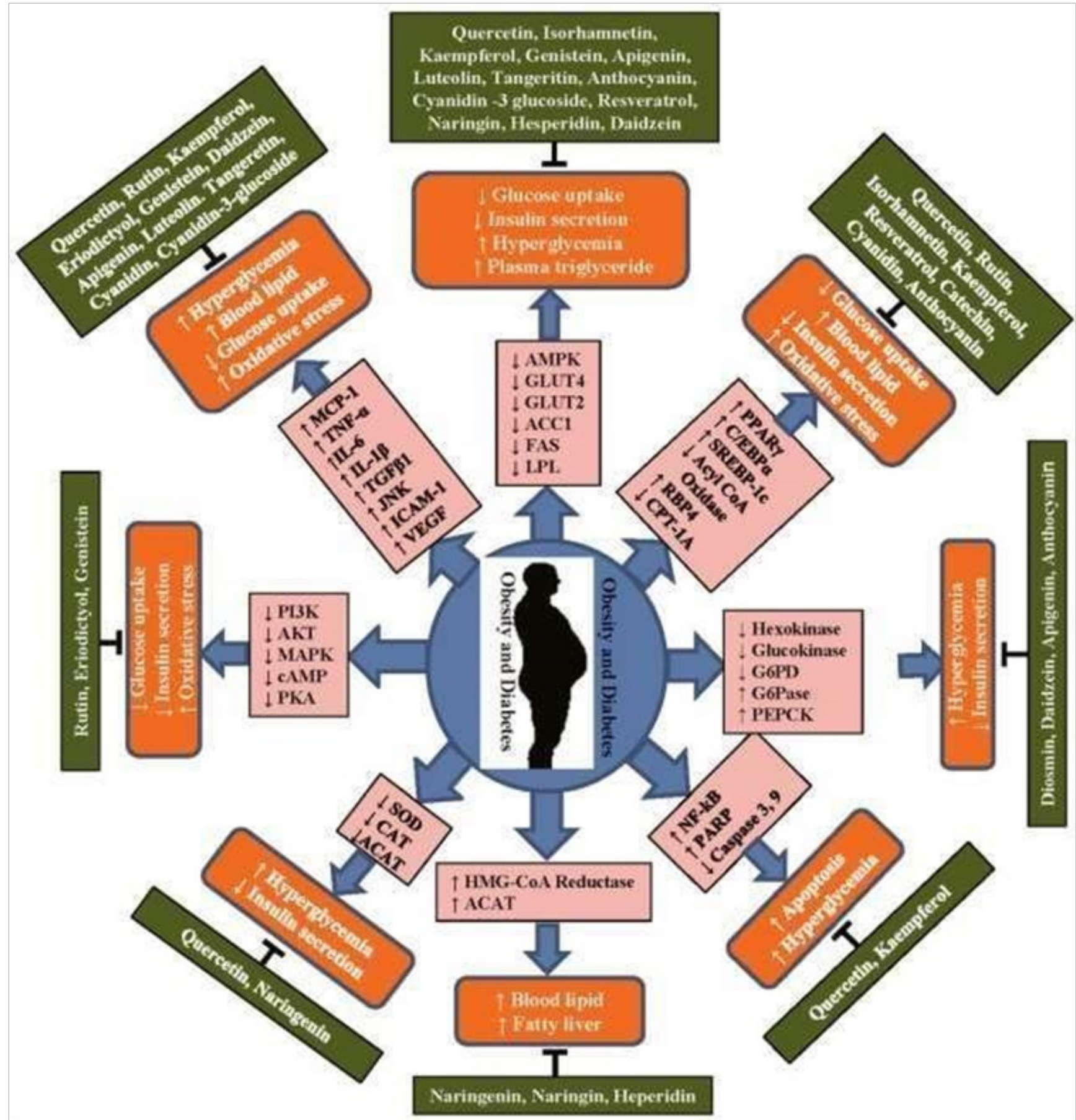

Figure 14 Schematic presentation of molecular functions of different flavonoids with anti-obesity and antidiabetic effects.

Flavonoids showed anti-obesity and anti-diabetic effects by activating or inhibiting different cytokines, enzymes, and metabolites to prevent inflammation, oxidative stress, and metabolism to protect against obesity and diabetes. MCP-I, monocyte-chemo-attractant protein-I;TNF- $\alpha$, tumor necrosis factor alpha; IL- 6 , interleukin-6; IL-I $\beta$, interleukin I beta; FFA, free fatty acid, IRSI, insulin receptor substrate I; PI3K, phosphatidylinositol 3-kinase;AKT, serine/threonine kinase; FA, fatty acid; IGT, impaired glucose tolerance; PARP, poly(ADP-ribose) polymerase; BCl-2, B-cell lymphoma 2; Bax, Bcl-2-associated X protein; Bak, Bcl-2 homologous antagonist/killer; Caspase 3, cysteine-dependent aspartate-directed proteases 3; PPAR $\gamma$, peroxisomal proliferatoractivated receptor gamma; SREBPIc, sterol regulatory element binding protein-Ic; LPL, lipo protein lipase; AMPK, 5' adenosine monophosphate-activated protein kinase; HOMA-IR, homeostatic model assessment for insulin resistance; HbA Ic, hemoglobin Alc; GLUT4, glucose transporter 4; G6PDH, glucose-6-phosphate dehydrogenase; HMG-CoA, 3-hydroxy3-methylglutaryl-coenzyme; ACAT, acyl CoA: cholesterol acyltransferase; G6pase, glucose6-phosphatase; cAMP, cyclic adenosine monophosphate; PKA, protein kinase A. 


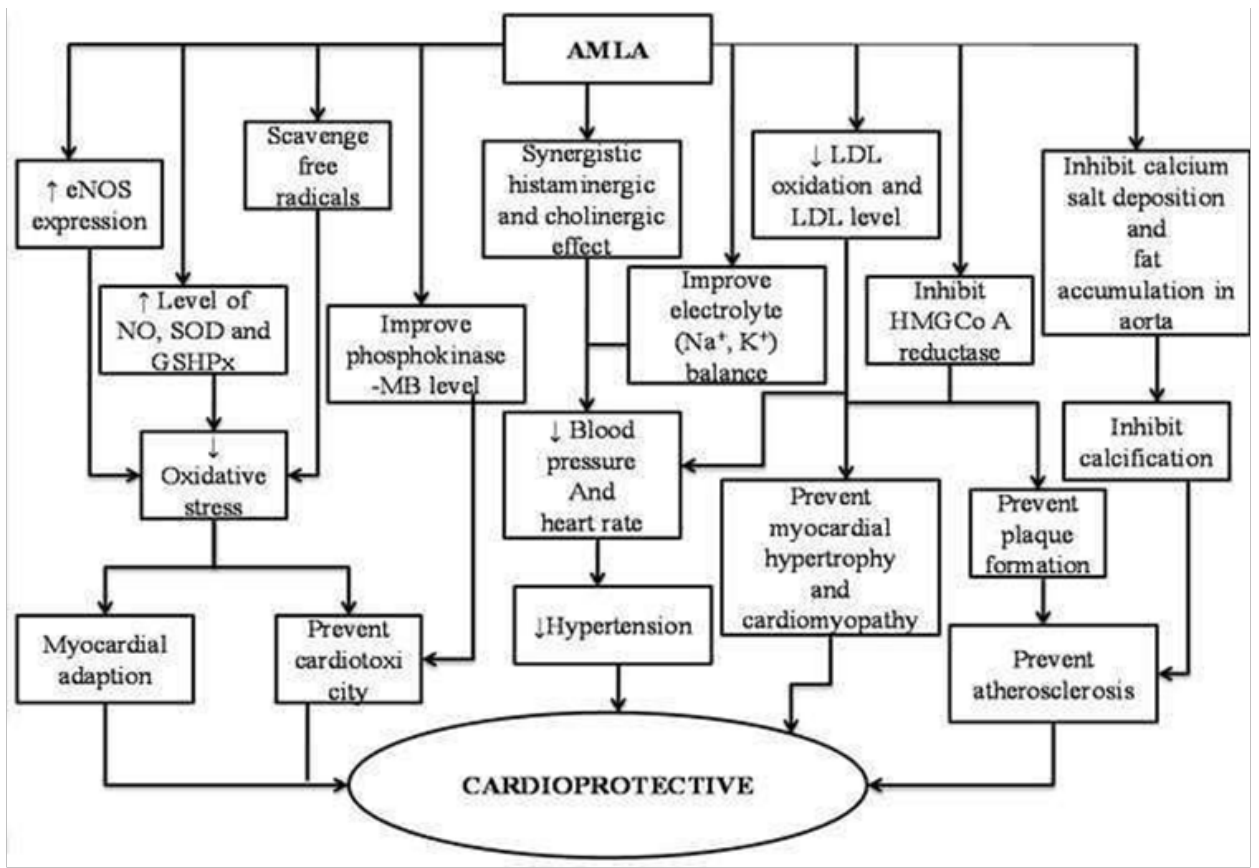

Figure 15 Mechanism of Amla as cardioprotective agent

(I) Reduction in hypertension and oxidative stress by increasing the expression of eNOS, by ameliorating the levels of nitric oxide (NO), SOD, GSHPx and electrolytes like $\mathrm{Na}+$ and $\mathrm{K}+$. (2) Aqueous extract of amla lowers the mean arterial blood pressure, heart rate and respiratory rate by showing synergistic histaminergic and cholinergic effect. (3) Cardioprotective potential in isoproterenol-induced cardiotoxicity through ameliorating the levels of antioxidant enzymes and creatinine phosphokinase-MB and LDH by its antioxidant free radical scavenging activity. (4) Amla fruit juice at dose of I ml/kg p.o for 8 weeks in diabetesinduced $\mathrm{Ml}$ showed preventive action in myocardial hypertrophy, cardiomyopathy and hypertension by increasing heart rate, lowering blood pressure through its antioxidant potential and by maintaining the lipid profile and enzyme levels. (5) Methanolic extract and fruit powder of amla has been known to attenuate the induction and progression of atherosclerosis by preventing the formation of plaque in blood vessels and aorta via inhibiting $\mathrm{HMG}$ CoA reductase and LDL oxidation; and by reducing LDL cholesterol levels and subsequently increasing HDL levels.

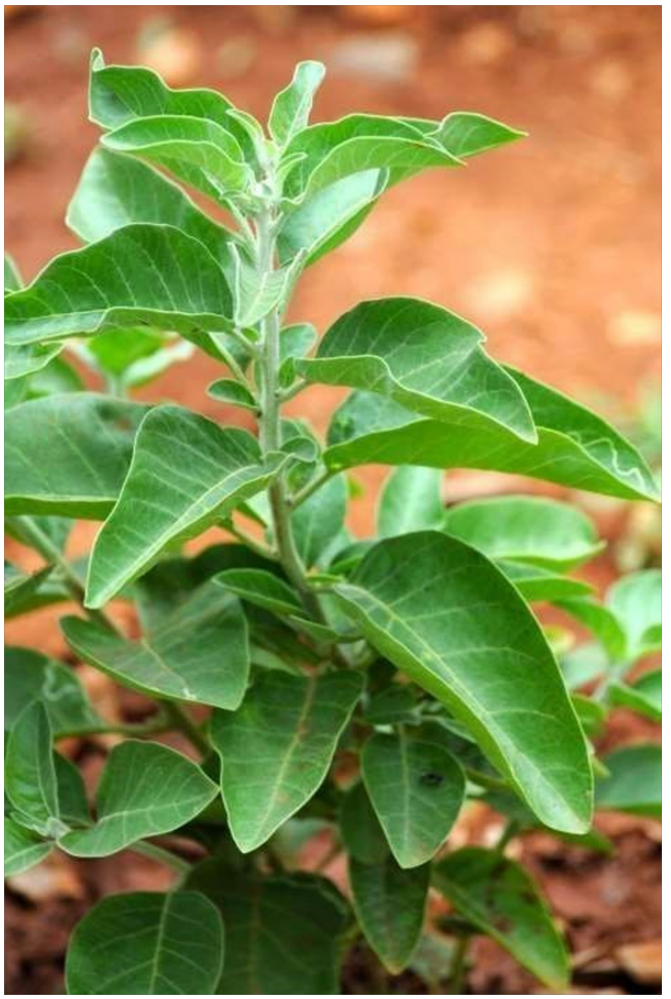

Figure 16 Ashwagandha (Withania somnifera) (Source: Red Moon Herbs)

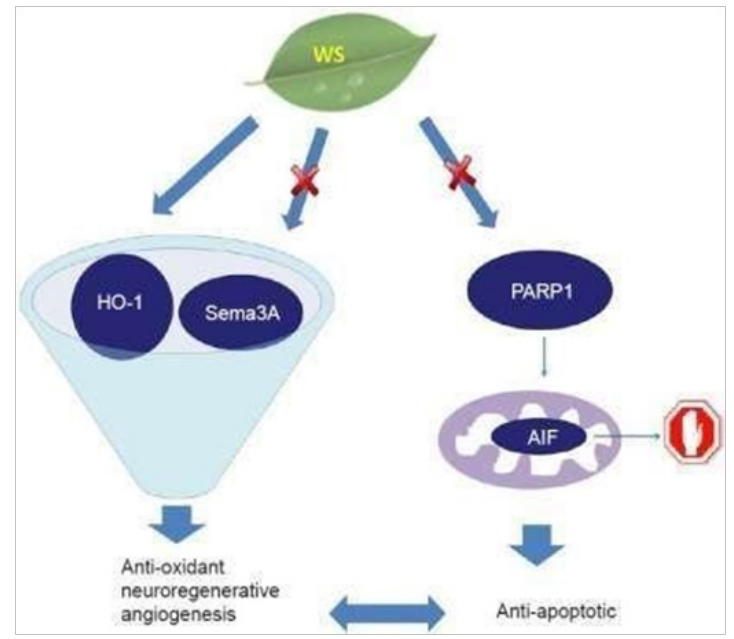

Figure 17 Probable mechanisms of action of an aqueous extract of Withania somnifera (WS) in ischemic stroke.

WS mediated attenuation of the expression of Semaphorin 3A (Sema3A) could promote neuronal regeneration. Moreover, $\mathrm{HO}-\mathrm{I}$ mediated vascular endothelial growth factor (VEGF) induction and the antagonistic effects of Sema3A and VEGF could have the combined result of higher VEGF levels and a resulting pro-angiogenic effect. WS was also found to reduce levels of poly (ADP-ribose) polymerase I (PARPI), which prevents translocation of antiapoptotic factor (AIF) from the mitochondria to the nucleus. The PARPIAIF pathway is a prime mediator of caspase independent apoptosis, which is prevented by WS in this model of stroke. 


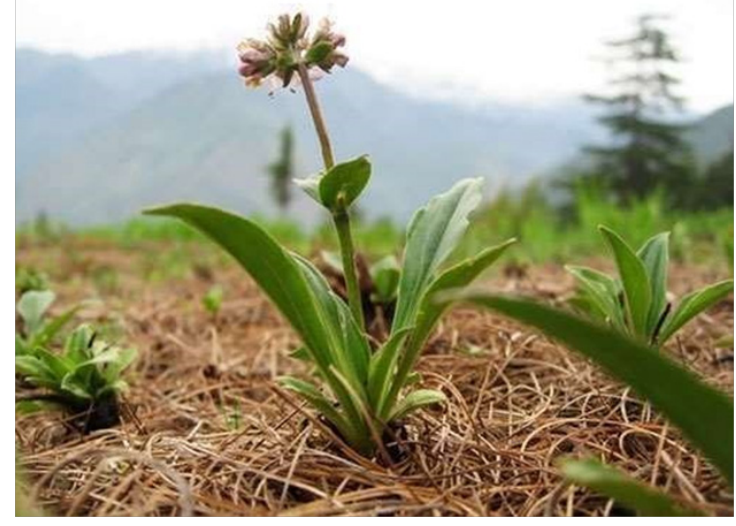

Figure I 8 Nardostachys jatamansi or spikenard (Source: fragnatica.com).

\section{Triphala (combination drug)}

An all-around perceived, polyherbal Ayurvedic medicine comprising of products of the plant species Emblica officinalis (Amalaki), Terminalia bellerica (Bibhitaki), and Terminalia chebula (Haritaki) in equivalent extent $1: 1: 1{ }^{154}$ Conceivably successful for a few clinical uses, for example, craving incitement, decrease of hyperacidity, cancer prevention agent, mitigating, immunomodulating, antibacterial, antimutagenic, adaptogenic, hypoglycemic, antineoplastic, chemoprotective, and radioprotective impacts, and avoidance of dental caries. ${ }^{155}$ Laghu shankha prakshalana kriya (LSP), a yogic bowel purifying strategy, coordinated methodology of yoga treatment (IAYT) is a protected and valuable system for patients with essential hypertension. LSP with triphala is progressively helpful. ${ }^{156}$ Assumes an essential job in pulse control and parities cholesterol. ${ }^{157,158}$ Triphala and its constituents can counter the impacts of an environment (ie, high dietary admission of fats) and have the potential for use as antiobesity operators with alluring lipid-profile balancing properties. ${ }^{159}$ Terminalia bellerica may impact cholesterol level for example increment the dimension of HDL and abatement LDL, and at the same time be valuable in the treatment of coronary supply route malady. ${ }^{160} \beta$-sitosterol, which is the principle constituent of T. chebula, is an organic compound having a place with the group of phytosterols, whose synthetic structure takes after cholesterol. $\beta$-sitosterol and phytosterols may influence lipid digestion by restraining the retention of cholesterol from the digestive tract. $\beta$-sitosterol has likewise been concentrated for its potential in bringing down large amounts of bloodcholesterol. ${ }^{161}$ Triphala detailing was related with hypolipidemic impacts on the tentatively actuated hypercholesteremic rodents. ${ }^{162}$ Triphala tablets help enhances the course and is a successful equation for hypercholesterolemia (Figure 19-21). ${ }^{163}$

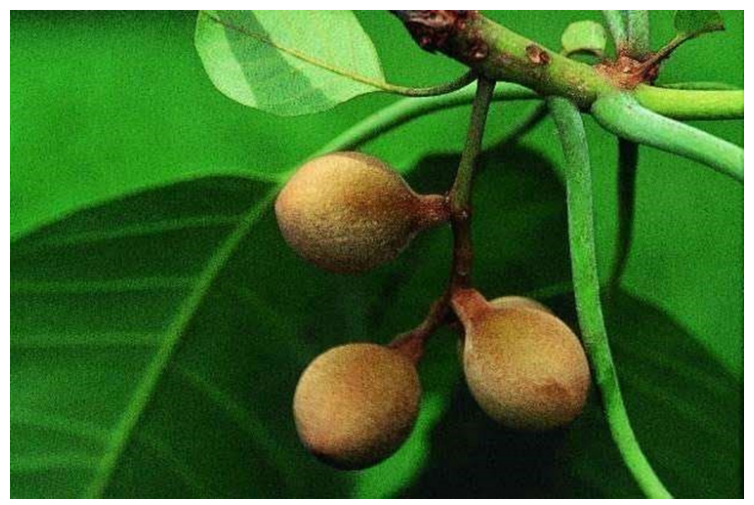

Figure 19 Terminalia bellerica (Bahera) (Source:Alibaba.com).

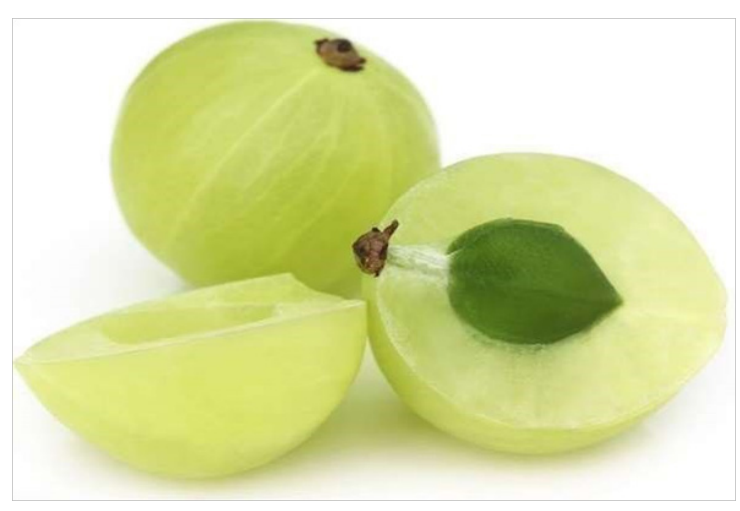

Figure 20 Phyllanthus Emblica (Amla) (Source:Ayurtimes.com).

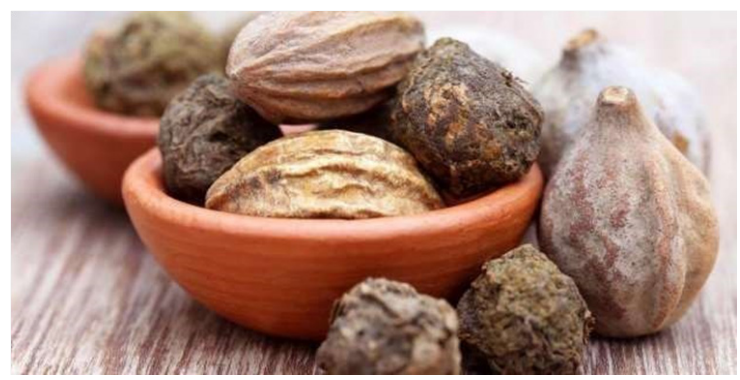

Figure 2I Terminalia chebula (Haritaki) (Source:AliExpress.com).

\section{Boerhavia diffusa (Nyctaginaceae)}

Punarnava, a notable cardiotonic eatable medicinal plant against apoptosis in Angiotensin II (Ang II)- invigorated hypertrophic cardiac cells (H9c2), powerful in lessening apoptosis in cardiac cells, which is a noteworthy supporter of sudden cardiac demise notwithstanding its nutraceutical properties. ${ }^{164}$ Different dynamic compounds in B. diffusa incorporate punarnavine, ursolic acid, punarnavoside, liriodendrin, eupalitin, eupalitin-3-O-â-D-galactopyranoside, rotenoids like boeravinones A, B, C, D, E, F and G, quercetin, kaempferol, and so forth. ${ }^{165,166}$ Among these, quercetin shows cell reinforcement, antihypertrophic and antihypertensive potential in vitro and in vivo test models. ${ }^{167,168}$ Ursolic acid is accounted for to have cardioprotective potential through initiating uncoupling of mitochondrial oxidative phosphorylation and lessening mitochondrial $\mathrm{H}_{2} \mathrm{O}_{2}$ generation. ${ }^{169}$ Kaempferol is additionally answered to have cardioprotective potential and boeravinone $\mathrm{G}$ is another cancer prevention agent and genoprotective compound in $\mathrm{B}$. diffusa. ${ }^{170,171}$ Liriodendrin segregated from B.diffusa is accounted for to have $\mathrm{Ca}^{2+}$ divert hostile properties in heart. ${ }^{172}$ Nearness of these dynamic constituents may be in charge of its defensive action against Ang II instigated hypertrophy. Cell organelles are likewise the objectives of Arsenic trioxide (ATO)initiated cardiotoxicity notwithstanding other detailed targets like particle channels, and ethanolic concentrate of Boerhavia diffusa can possibly ensure the cardiotoxicity incited by ATO (Figure 22). ${ }^{173}$

\section{Cruciferous vegetables}

This incorporate broccoli, Brussel grows cabbage, cauliflower, kale, radish, rutabaga, turnip and even arugula. cruciferous vegetables contain isothiocyanates, which can create the redoxcontrolled cardioprotective protein, thioredoxin, it was contemplated that utilization of broccoli could be advantageous to the heart. Sulforaphane is by a wide margin the most generally concentrated and best described isothiocyanates. ${ }^{174,175}$ Research is demonstrating that sulforaphane assists with irritation of the blood vessel dividers, restrains corpulence, diminishes hypertension, and different 
conditions that are a piece of or lead to CVD. ${ }^{176}$ Oxidative pressure brought about by diminished generation of nitric oxide as well as expanded creation of ROS (principally superoxide) may advance endothelial brokenness. Accordingly, expanded oxidative pressure speaks to one conceivable driver of the expanded predominance of hypertension. An eating routine containing broccoli grows high in Grn (Grn+) diminished oxidative pressure and related issues in male immediately hypertensive stroke-inclined rodents (SHRsp). ${ }^{177}$ As one of Grn key metabolites, SFN was additionally found to enhance pulse. Weight is related with metabolic turmoil, which is another hazard factor for atherosclerosis and CVD. Choi et al. examined whether SFN counteracted high-fat eating regimen (HFD-) instigated corpulence in C57BL/6N mice. SFN may actuate hostile to corpulence impacts by restraining adipogenesis. ${ }^{178} 75 \%$ of the sulforaphane will be retained into the circulation system after gobbled and taken up by cells. Once inside sulforaphane can harm essential intracellular structures like mitochondria and compounds. This drains glutathione (most powerful human cancer prevention agent) leaving cells defenseless against further oxidative harm. Sulforaphane can even disturb epithelial hindrances giving one more plant concoction that can add to "flawed gut (Figure 23)."179

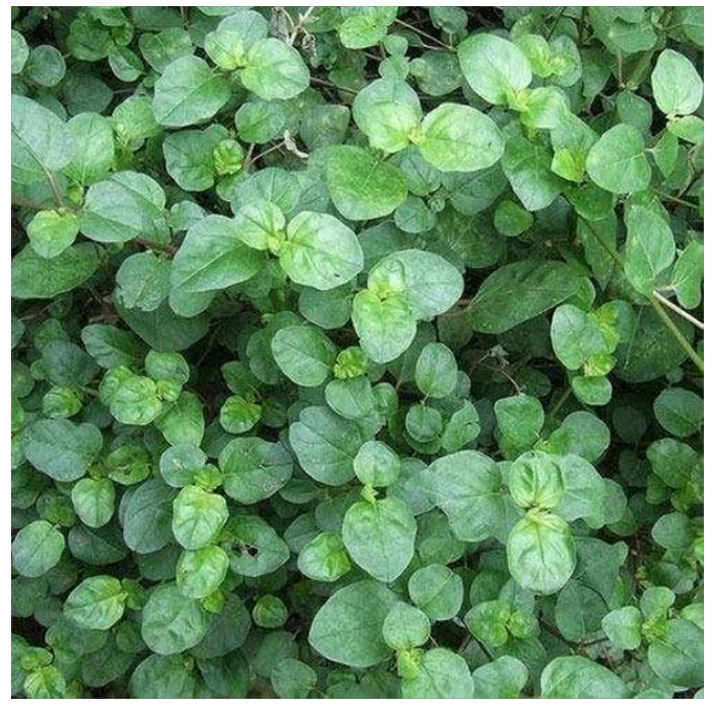

Figure 22 Boerhavia diffusa (Nyctaginaceae), Punarnava (Source: Indiamart).

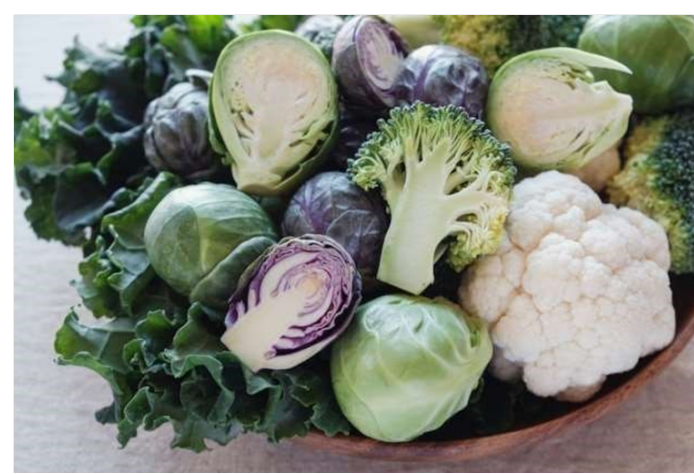

Figure 23 Cruciferous vegetables (Source: roswellpark.org).

\section{Nigella sativa (Ranunculaceae)}

It has been utilized in traditional medicine and a few investigations have been performed in the most recent decades to uncover the impacts of it on various therapeutic issue, for example, diabetes, dyslipidemia, hypertension, and stoutness. ${ }^{180} \mathrm{~N}$. sativa oil at a portion of $2.5 \mathrm{mg} /$ $\mathrm{kg}$ constricts the N $\omega$-nitro-L-arginine methyl ester (L-NAME)instigated increment in BP and was related with a decrease in cardiac redox status and angiotensin-changing over catalyst movement and an expansion in heme oxygenase (HO-1) action. N. sativa oil additionally anticipated plasma NO misfortune. Remarkably, the BP-bringing impact was similar down to that of nicardipine in hypertensive rodents. ${ }^{181} \mathrm{~N}$. sativa and its segment thymoquinone have the helpful impact on hypertension most likely because of weakening cardiovascular impacts of angiotensin II. ${ }^{182}$ Thymoquinone (the fundamental constituent Nigella sativa) supplementation lessens cyclophosphamide-actuated cardiotoxicity, at any rate partially, by its capacity to diminish oxidative and nitrosative pressure and to save the movement of cancer prevention agent catalysts just as its capacity to enhance the mitochondrial capacity and vitality creation. ${ }^{183} \mathrm{~A}$ comparative report was found with cyclosporine An and Doxorubicin. N. sativa oil diminished the resulting cyclosporine damage in rodent heart, exhibited by standardized cardiac histopathology, decline in lipid peroxidation, and enhancement in cancer prevention agent catalyst status and cell protein oxidation. ${ }^{184}$ Thymoquinone as a possibly particular cytoprotective operator, which may enhance cardiotoxicity without diminishing DOX antitumor movement (Figure 24). ${ }^{185}$

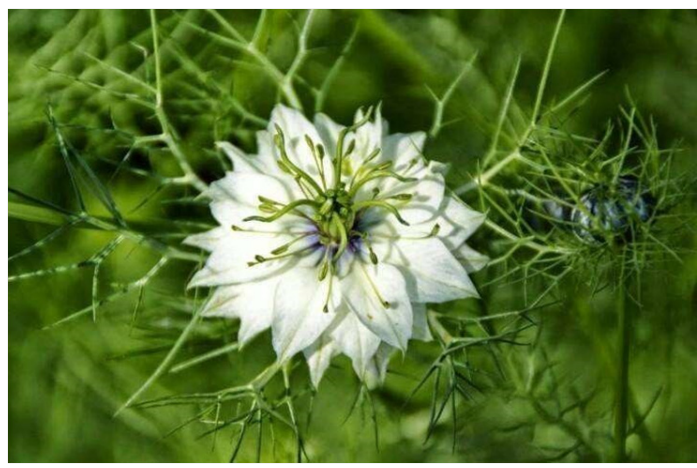

Figure 24 Nigella sativa (black cumin) (Source: Healthy Buddha Magazine).

\section{Zingiber officinale (Zingiberaceae)}

The pharmacological exercises of ginger were mostly ascribed to its dynamic phyto compounds 6-gingerol, 6-shogaol, zingerone adjacent to different phenolics and flavonoids. 6-gingerol was accounted for as the most plentiful bioactive compound in ginger with different pharmacological impacts including cell reinforcement, pain relieving, mitigating and antipyretic properties. Likewise, different investigations demonstrated that 6-shogaol with most reduced fixation in ginger speak to all the more naturally actives contrasted with 6-gingerol. As per creature ponders ginger can possibly offer a characteristic elective dietary supplementation to traditional enemy of hypertensive operators, yet increasingly human preliminaries of ginger on hypertensive patients utilizing diverse measurements of an institutionalized concentrate are required. ${ }^{186}$ The antihyperlipidemic impact of ginger was bolstered by creature examines, gingerol forestalls HFD-incited hyperlipidemia by regulating the outflow of compounds critical to cholesterol digestion. ${ }^{187}$ Also, the bringing down impact of ginger on serum cholesterol might be because of the inhibitory impact of cholesterol biosynthesis and the change of cholesterol into bile acids by raising the movement of hepatic cholesterol 7 alpha-hydroxylase. Moreover, niacin, a supplement in ginger, might be a potential dynamic fixing in bringing down serum triglyceride level, expanding leeway of VLDL, improving hepatic take-up of LDL-c, and hindering cholesterol combination. ${ }^{188}$ Late 
investigation appears, 6-gingerol could diminish cell all out cholesterol and free cholesterol levels by means of up-direction of LDLR through initiation of SREBP2 just as up-control of cholesterol efflux-related qualities LXR $\alpha$ and ABCA1 (part 1 of human transporter subfamily ABCA). ${ }^{189}$ Day by day organization of $1 \mathrm{~g}$ ginger diminishes serum triglyceride fixation, which is a hazard factor for cardiovascular illness, in peritoneal dialysis patients (Figure 25). ${ }^{190,191}$

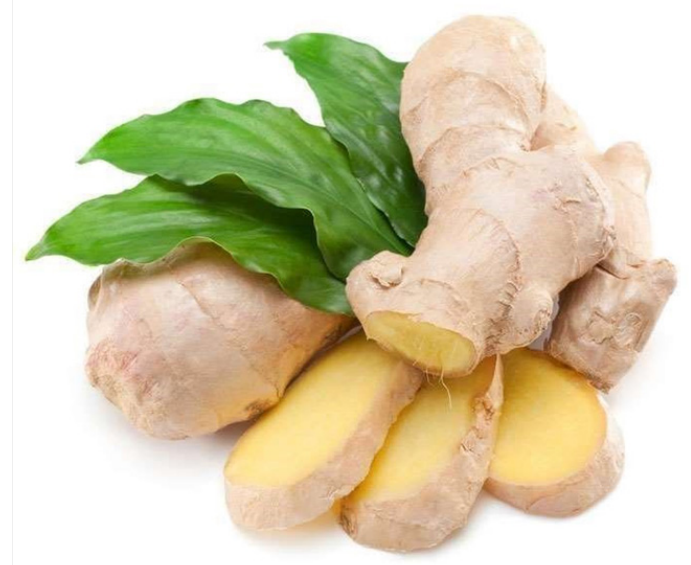

Figure 25 Zingiber officinale (Zingiberaceae) Rhizome (Source:WordPress com)

\section{Conclusion}

The pharmacological activities of herbs or herbal segregates appear to positively regulate a few parameters ensnared in the pathogenesis of pulse, including however not restricted to ROS creation, VSMC phenotype, endothelial capacity, platelet initiation, star inflammatory flagging, and quality articulation. With such an expansive range of activities, one may anticipate that herbal cures will get considerably more consideration in the coming years, maybe emphasizing the requirement for further experimentations and clinical preliminaries. To be sure, the absence of adequate clinical preliminaries establishes a noteworthy impediment on the ir use right now. Of equivalent significance, it might be prudent that patients be fittingly taught, especially in connection to herbs whose utilization has been viewed as safe for a huge number of years (dark cumin, Chinese sage, coriander, garlic, ginger, ginseng, and tea), and has been bolstered by sound logical proof, for example, one dependent on clinical preliminaries with substantial populace gatherings. The recharged enthusiasm for the scan for new medications from characteristic sources, particularly from plant sources, has increased worldwide consideration amid the most recent two decades. The tropical downpour woods have turned into an essential purpose of this movement, principally because of the rich biodiversity they harbor, which guarantees a high decent variety of chemicals with the potential novel structures. Be that as it may, of this rich biodiversity, just a little segment has been concentrated for its medicinal potential. The proof exhibited is emphatically characteristic of the idea that herbs and plants are ending up some portion of proof based medicine in the avoidance and additionally treatment of CVD.

\section{Acknowledgments}

None.

\section{Conflicts of interest}

Author declares that there is no conflicts of interest.

\section{References}

1. Jin J. Screening for Cardiovascular Disease Risk with ECG. JAMA. 2018;319(22):2346.

2. Cholesterol and Atherosclerosis. JAMA. 2001;285(19):2536.

3. Kronhaus KD. Dysrhythmia vs Arrhythmia. JAMA. 1979;241(1):28.

4. White PD. An Unusual Type Of Gross Cardiac Arrhythmia. JAMA. 1915;LXV(15):1276.

5. Cardiac Arrhythmias: Diagnosis and Treatment. Arch Intern Med.1977;137(11):1643.

6. Caselli S, Serdoz A, Mango F, et al. High blood pressure response to exercise predicts future development of hypertension in young athletes. Eur Heart J. 2019;40(1):62-68.

7. Kristian Thygesen, Joseph S Alpert, Allan S Jaffe, et al. Fourth universal definition of myocardial infarction (2018). Eur Heart J. 2019;40(3):237269.

8. Zhao-Jun Yang, Jie Liu, Jia-Pu Ge, et al. Prevalence of cardiovascular disease risk factor in the Chinese population: the 2007-2008 China National Diabetes and Metabolic Disorders Study. Eur Heart J. 2012;33(2):213-220.

9. Jin J. Risk Assessment for Cardiovascular Disease with Nontraditional Risk Factors. JAMA. 2018;320(3):316.

10. Murabito JM, Guo CY, Fox CS, et al. Heritability of the AnkleBrachial Index: The Framingham Offspring Study. Am J Epidemiol. 2006;164(10):963-968.

11. Satoh K, Shimokawa H. High-sensitivity C-reactive protein: still need for nextgeneration biomarkers for remote future cardiovascular events, Eur Heart J. 2014;35(27):1776-1778.

12. Tota-Maharaj R, Blaha MJ, McEvoy JW, et al. Coronary artery calcium for the prediction of mortality in young adults $<45$ years old and elderly adults >75 years old. Eur Heart J. 2012;33(23):2955-2962.

13. Shahi MSJR, Dey M, Chowdhury AK. 20P A study on possibility of high sensitivity C- reactive protein (hs-CRP) and circulating interluekin-6 (IL-6) as biomarker in breast cancer patients. Annals of Oncology. 2018;29(suppl_9).

14. Kotecha D, Piccini JP. Atrial fibrillation in heart failure: what should we do? Eur Heart J. 2015;36(46):3250-3257.

15. Aidan P Bolger, Andrew JS Coats, Michael A Gatzoulis. Congenital heart disease: the original heart failure syndrome. Eur Heart J. 2003;24(10):970-976.

16. Thomas F Lüscher. Outcome of congenital heart disease with modern cardiac care. European Heart Journal. 2018;39(12):969-971.

17. Fukui M, Gupta A, Abdelkarim I, et al. Association of Structural and Functional Cardiac Changes With Transcatheter Aortic Valve Replacement Outcomes in Patients With Aortic Stenosis. JAMA Cardiol; 2019.

18. Maron BJ, Lesser JR, Schiller NB, et al. Implications of Hypertrophic Cardiomyopathy Transmitted by Sperm Donation. JAMA. 2009;302(15):1681-1684.

19. Angus DC. Successful Resuscitation From In-Hospital Cardiac ArrestWhat Happens Next? JAMA. 2015;314(12):1238-1239.

20. Myerburg RJ, Goldberger JJ. Sudden Cardiac Arrest Risk Assessment: Population Science and the Individual Risk Mandate. JAMA Cardiol. 2017;2(6):689-694.

21. Hess CN, Hiatt WR. Antithrombotic Therapy for Peripheral Artery Disease in 2018. JAMA. 2018;319(22):2329-2330. 
22. Voelker R. Aneurysm Guidelines. JAMA. 2000;284(20):2585.

23. Dan Laukka, Emily Pan, Terhi Fordell, et al. 317 Prevalence of Thoracic Aneurysms or Dilatations in Patients With the Intracranial Aneurysms. Neurosurgery. 2018;65(suppl_1):128.

24. Mehmet Kanbay, Laura-Gabriela Sánchez-Lozada, Martha Franco, et al. Microvascular disease and its role in the brain and cardiovascular system: a potential role for uric acid as a cardiorenal toxin. Nephrol Dial Transplant. 2011;26(2):430-437.

25. Coccheri S, Palareti G. The cardiovascular risk burden of intermittent claudication. European Heart Journal Supplements. 2002;4(suppl_B):B46-B49.

26. Bell CS, Samuel JP, Samuels JA. Prevalence of Hypertension in Children. Hypertension. 2019;73(1):148-152.

27. Anker D, Santos-Eggimann B, Santschi V, et al. Screening and treatment of hypertension in older adults: less is more? Public Health Rev. 2018;39:26.

28. Kim H, Andrade FCD. Diagnostic status and age at diagnosis of hypertension on adherence to lifestyle recommendations. Prev Med Rep. 2018;13:52-56.

29. Moynihan RN, Clark J, Albarqouni L. Media Coverage of the Benefits and Harms of the 2017 Expanded Definition of High Blood Pressure. JAMA Intern Med. 2019;179(2):272-273.

30. Roth GA, Johnson CO, Abate KH, et al. The Burden of Cardiovascular Diseases Among US States, 1990-2016. JAMA Cardiol. 2018;3(5):375389.

31. Khera R, Lu Y, Lu J, et al. Impact of 2017 ACC/AHA guidelines on prevalence of hypertension and eligibility for antihypertensive treatment in United States and China: nationally representative cross sectional study. BMJ. 2018;362:k2357.

32. Wyndham CR. Atrial fibrillation: the most common arrhythmia. Tex Heart Inst J. 2000;27(3):257-267.

33. Zoni-Berisso M, Lercari F, Carazza T, et al. Epidemiology of atrial fibrillation: European perspective. Clin Epidemiol. 2014;6:213-220.

34. Ko D, Rahman F, Schnabel RB, et al. Atrial fibrillation in women: epidemiology, pathophysiology, presentation, and prognosis. Nat Rev Cardiol. 2016;13(6):321-332.

35. Bradley SM, Borgerding JA, Wood GB, et al. Incidence, Risk Factors, and Outcomes Associated With In-Hospital Acute Myocardial Infarction. JAMA Netw Open. 2019;2(1):e187348.

36. Kayani WT, Ballantyne CM. Improving Outcomes After Myocardia Infarction in the US Population. J Am Heart Assoc. 2018;7(4):e008407.

37. Kim J, Thrift AG. A Promising Skills-Based Intervention to Reduce Blood Pressure in Individuals with Stroke and Transient Ischemic Attack. JAMA Neurol. 2019;76(1):13-14.

38. Sanchis-Gomar F, Perez-Quilis C, Leischik R, et al. Epidemiology of coronary heart disease and acute coronary syndrome. Ann Transl Med. 2016;4(13):256.

39. Mokdad AH, Ballestros K, Echko M, et al. The State of US Health, 19902016: Burden of Diseases, Injuries, and Risk Factors Among US States. JAMA. 2018;319(14):1444-1472.

40. Shaw LJ, Goyal A, Mehta C, et al. 10-Year Resource Utilization and Costs for Cardiovascular Care. J Am Coll Cardiol. 2018;71(10):10781089.

41. Gheorghe A, Griffiths U, Murphy A, et al. The economic burden of cardiovascular disease and hypertension in low- and middleincome countries: a systematic review. BMC Public Health. 2018;18(1):975.
42. Yu E, Malik VS, Hu FB. Cardiovascular Disease Prevention by Diet Modification: JACC Health Promotion Series. J Am Coll Cardiol. 2018;72(8):914-926.

43. Billingsley HE, Carbone S, Lavie CJ. Dietary Fats and Chronic Noncommunicable Diseases. Nutrients. 2018;10(10):E1385.

44. Prentice RL, Aragaki AK, Van Horn L, et al. Low-fat dietary pattern and cardiovascular disease: results from the Women's Health Initiative randomized controlled trial. Am J Clin Nutr. 2017;106(1):35-43.

45. Forouhi NG, Krauss RM, Taubes G, et al. Dietary fat and cardiometabolic health: evidence, controversies, and consensus for guidance. BMJ. 2018;361:k2139.

46. Oh R, Uppaluri KR. Low Carbohydrate Diet. Treasure Island (FL): StatPearls Publishing; 2018.

47. Seidelmann SB, Claggett B, Cheng S, et al. Dietary carbohydrate intake and mortality: a prospective cohort study and meta-analysis. Lancet Public Health. 2018;3(9):e419-e428.

48. Vitale M, Masulli M, Calabrese I, et al. Impact of a Mediterranean Dietary Pattern and Its Components on Cardiovascular Risk Factors, Glucose Control, and Body Weight in People with Type 2 Diabetes: A Real-Life Study. Nutrients. 2018;10(8):E1067.

49. Lim CC, Hayes RB, Ahn J, et al. Mediterranean Diet and the Association between Air Pollution and Cardiovascular Disease Mortality Risk. Circulation; 2019.

50. Eilat-Adar S, Sinai T, Yosefy C, et al. Nutritional recommendations for cardiovascular disease prevention. Nutrients. 2013;5(9):3646-3683.

51. Zhao CN, Meng X, Li Y, et al. Fruits for Prevention and Treatment of Cardiovascular Diseases. Nutrients. 2017;9(6):E598.

52. Bonaccio M, Di Castelnuovo A, Costanzo S, et al. Moli-sani Study Investigators. Interaction between Mediterranean diet and statins on mortality risk in patients with cardiovascular disease: Findings from the Moli-sani Study. Int J Cardiol. 2019;276:248-254.

53. Kapil V, Khambata RS, Robertson A, et al. Dietary nitrate provides sustained blood pressure lowering in hypertensive patients: a randomized, phase 2, double-blind, placebo-controlled study. Hypertension. 2015;65(2):320-327.

54. Bazzano LA. Effects of soluble dietary fiber on low-density lipoprotein cholesterol and coronary heart disease risk. Curr Atheroscler Rep. 2008;10(6):473-477.

55. Zafra-Stone S, Yasmin T, Bagchi M, et al. Berry anthocyanins as novel antioxidants in human health and disease prevention. Mol Nutr Food Res. 2007;51(6):675-683.

56. Wang L, Bordi PL, Fleming JA, et al. Effect of a moderate fat diet with and without avocados on lipoprotein particle number, size and subclasses in overweight and obese adults: a randomized, controlled trial. $\mathrm{J} \mathrm{Am}$ Heart Assoc. 2015;4(1):e01355.

57. Hernández-Alonso P, Bulló M, Salas-Salvadó J. Pistachios for Health: What Do We Know About This Multifaceted Nut? Nutr Today. 2016;51(3):133-138.

58. Galleano M, Oteiza PI, Fraga CG. Cocoa, chocolate, and cardiovascular disease. J Cardiovasc Pharmacol. 2009;54(6):483-490.

59. Story EN, Kopec RE, Schwartz SJ, et al. An update on the health effects of tomato lycopene. Annu Rev Food Sci Technol. 2010;1:189-210.

60. Ros E. Health benefits of nut consumption. Nutrients. 2010;2(7):652682.

61. Gammone MA, Riccioni G, D'Orazio N. Carotenoids: potential allies of cardiovascular health? Food Nutr Res. 2015;59:26762. 
62. Kishimoto $\mathrm{Y}$, Yoshida $\mathrm{H}$, Kondo $\mathrm{K}$. Potential Anti-Atherosclerotic Properties of Astaxanthin. Mar Drugs. 2016;14(2):E35.

63. Fassett RG, Coombes JS. Astaxanthin: a potential therapeutic agent in cardiovascular disease. Mar Drugs. 2011;9(3):447-465.

64. Aoi W, Naito Y, Sakuma K, et al. Astaxanthin limits exercise-induced skeletal and cardiac muscle damage in mice. Antioxid Redox Signal. 2003;5(1):139-144.

65. Ambati RR, Phang SM, Ravi S, et al. Astaxanthin: sources, extraction, stability, biological activities and its commercial applications-a review. Mar Drugs. 2014;12(1):128-152.

66. Heo SJ, Yoon WJ, Kim KN, et al. Evaluation of anti-inflammatory effect of fucoxanthin isolated from brown algae in lipopolysaccharidestimulated RAW 264.7 macrophages. Food Chem Toxicol. 2010;48(89):2045-2451

67. Chang PM, Li KL, Lin YC. Fucoidan-Fucoxanthin Ameliorated Cardiac Function via IRS1/GRB2/SOS1, GSK3 $\beta /$ CREB Pathways and Metabolic Pathways in Senescent Mice. Mar Drugs. 2019;17(1):E69.

68. Zhang H, Tang Y, Zhang Y, et al. Fucoxanthin: A Promising Medicina and Nutritional Ingredient. Evid Based Complement Alternat Med. 2015;2015:723515.

69. Maeda H, Kanno S, Kodate M, et al. Fucoxanthinol, Metabolite of Fucoxanthin, Improves Obesity-Induced Inflammation in Adipocyte Cells. Mar Drugs. 2015;13(8):4799-4813.

70. Cheng HM, Koutsidis G, Lodge JK, et al. Lycopene and tomato and risk of cardiovascular diseases: A systematic review and meta-analysis of epidemiological evidence. Crit Rev Food Sci Nutr. 2017;59(1):141-158.

71. Zou ZY, Xu XR, Lin XM, et al. Effects of lutein and lycopene on carotid intima-media thickness in Chinese subjects with subclinical atherosclerosis: a randomised, double-blind, placebo-controlled trial. $\mathrm{Br}$ J Nutr. 2014;111(3):474-480.

72. Mozos I, Stoian D, Caraba A, et al. Lycopene and Vascular Health. Front Pharmacol. 2018;9:521.

73. Thies F, Mills LM, Moir S, et al. Cardiovascular benefits of lycopene fantasy or reality? Proc Nutr Soc. 2017;76(2):122-129.

74. Sesso HD, Liu S, Gaziano JM, et al. Dietary Lycopene, TomatoBased Food Products and Cardiovascular Disease in Women. $J$ Nutr. 2003;133(7):2336-2341.

75. Sommerburg O, Keunen JE, Bird AC, et al. Fruits and vegetables that are sources for lutein and zeaxanthin: the macular pigment in human eyes. $\mathrm{Br}$ J Ophthalmol. 1998;82(8):907-910.

76. Kim JE, Leite JO, DeOgburn R, et al. A luteinenriched diet prevents cholesterol accumulation and decreases oxidized LDL and inflammatory cytokines in the aorta of guinea pigs. J Nutr. 2011;141(8):1458-1463.

77. Thomson RL, Coates AM, Howe PR, et al. Increases in plasma lutein through supplementation are correlated with increases in physical activity and reductions in sedentary time in older adults. Nutrients. 2014;6(3):974-984.

78. Han H, Cui W, Wang L, et al. Lutein prevents high fat dietinduced atherosclerosis in ApoE-deficient mice by inhibiting NADPH oxidase and increasing PPAR expression. Lipids. 2015;50(3):261-273.

79. Viera I, Pérez-Gálvez A, Roca M. Bioaccessibility of Marine Carotenoids. Mar Drugs. 2018;16(10):E397.

80. Wang C, Qiu R, Cao Y, et al. Higher dietary and serum carotenoid levels are associated with lower carotid intima-media thickness in middle-aged and elderly people. Br J Nutr. 2018;119(5):590598.

81. Dwyer JH, Paul-Labrador MJ, Fan J, et al. Progression of carotid intimamedia thickness and plasma antioxidants: the Los Angeles Atherosclerosis Study. Arterioscler Thromb Vasc Biol. 2004;24(2):313-319.
82. Lutein and Zeaxanthin Linked to Higher Heart Attack Risk. Review of Optometry. 2005;142(8).

83. Burri BJ, La Frano MR, Zhu C. Absorption, metabolism, and functions of $\beta$-cryptoxanthin. Nutr Rev. 2016;74(2):69-82.

84. Pongkan $\mathrm{W}$, Takatori $\mathrm{O}, \mathrm{Ni} \mathrm{Y}$, et al. $\beta$-Cryptoxanthin exerts greater cardioprotective effects on cardiac ischemia-reperfusion injury than astaxanthin by attenuating mitochondrial dysfunction in mice. Mol Nutr Food Res. 2017;61(10)

85. Iribarren C, Folsom AR, Jacobs DR, et al. Association of serum vitamin levels, LDL susceptibility to oxida-tion, and autoantibodies againstMDALDL withcarotid atherosclerosis. A case-control study. The ARIC Study Investigators Atherosclerosis Risk in Communities. Arterioscler Thromb Vasc Biol. 1997;17(6):1171-1177.

86. Llopis S, Rodrigo MJ, González N, et al. $\beta$-Cryptoxanthin Reduces Body Fat and Increases Oxidative Stress Response in Caenorhabditis elegans Model. Nutrients. 2019;11(2):E232.

87. Nakamura M, Sugiura M, Aoki N. High beta-carotene and betacryptoxanthin are associated with low pulse wave velocity. Atherosclerosis. 2006;184(2):363-369.

88. Kritchevsky SB. $\beta$-Carotene, Carotenoids and the Prevention of Coronary Heart Disease. J Nutr. 1999;129(1):5-8.

89. Alija AJ, Bresgen N, Sommerburg O, et al. Cyto- and genotoxic potential of beta-carotene and cleavage products under oxidative stress. Biofactors. 2005;24(1-4):159-163.

90. Siems W, Salerno C, Crifo C, et al. Beta-carotene degradation productsformation, toxicity and prevention of toxicity. Forum Nutr. 2009;61:75-

91. Csepanyi E, Czompa A, Haines D, et al. Cardiovascular effects of low versus high-dose beta-carotene in a rat model. Pharmacol Res. 2015;100:148-156.

92. Zornoff LA, Matsubara LS, Matsubara BB, et al. Beta-Carotene Supplementation Attenuates Cardiac Remodeling Induced by One-Month Tobacco-Smoke Exposure in Rats. Toxicol Sci. 2006;90(1):259-266.

93. Voutilainen S, Nurmi T, Mursu J, et al. Carotenoids and cardiovascular health. Am J Clin Nutr. 2006;83(6):1265-1271.

94. Ganguly P, Alam SF. Role of homocysteine in the development of cardiovascular disease. Nutr J. 2015;14:6.

95. Hankey GJ, Eikelboom JW. Homocysteine and vascular disease. Lancet. 1999;354(9176):407-413.

96. Marcus J, Sarnak MJ, Menon V. Homocysteine lowering and cardiovascular disease risk: lost in translation. Can $J$ Cardiol. 2007;23(9):707-710

97. Fallis J. 16 Powerful Ways to Effectively Lower Homocysteine. Web Optimal Living Dynamics; 2017.

98. Nieva R, Safavynia SA, Lee Bishop K, et al. Herbal, vitamin, and minera supplement use in patients enrolled in a cardiac rehabilitation program. $J$ Cardiopulm Rehabil Prev. 2012;32(5):270-277.

99. Grassi D, Desideri G, Croce G, et al. Flavonoids, vascular function and cardiovascular protection. Curr Pharm Des. 2009;15(10):1072-1084.

100. Panche AN, Diwan AD, Chandra SR. Flavonoids: an overview. J Nutr Sci. 2016;5:e47.

101. Geleijnse JM, Launer LJ, Van der Kuip DA, et al. Inverse association of tea and flavonoid intakes with incident myocardial infarction: the Rotterdam Study. Am J Clin Nutr. 2002;75(5):880-886.

102. Arts IC, Hollman PC, Feskens EJ, et al. Catechin intake might explain the inverse relation between tea consumption and ischemic heart disease: the Zutphen Elderly Study. Am J Clin Nutr. 2001;74(2):227-232. 
103. Kurian G, Paddikkala J. Effect of IntraOperative Magnesium Supplementation in Plasma Antioxidant Levels Trace Elements and Electrolyte Balance in Serum of Coronary Artery Bypass Graft Patients. J Clin Basic Cardiol. 2007;10(1):11-15.

104. Muñoz-Castañeda JR, Pendón-Ruiz de Mier MV, Rodríguez M, et al. Magnesium Replacement to Protect Cardiovascular and Kidney Damage? Lack of Prospective Clinical Trials. Int J Mol Sci. 2018;19(3):E664.

105. Petre A. 21 Vegetarian Foods That Are Loaded With Iron. Web HealthLine; 2017.

106. Tadimalla RT. Top 39 Magnesium-Rich Foods You Should Include In Your Diet. Web Stylecraze; 2019.

107. Ordway GA, Garry DJ. Myoglobin: an essential hemoprotein in striated muscle. J Exp Biol. 2004;207(Pt 20):3441-3446.

108. Mordi IR, Tee A, Lang CC. Iron Therapy in Heart Failure: Ready for Primetime? Card Fail Rev. 2018;4(1):28-32.

109. Kshirsagar AV, Freburger JK, Ellis AR, et al. Intravenous iron supplementation practices and short-term risk of cardiovascular events in hemodialysis patients. PLoS One. 2013;8(11):e78930.

110. Bowen KJ, Harris WS, Kris-Etherton PM. Omega-3 Fatty Acids and Cardiovascular Disease: Are There Benefits? Curr Treat Options Cardiovasc Med. 2016;18(11):69.

111. Gammone MA, Riccioni G, Parrinello G, et al. Omega-3 Polyunsaturated Fatty Acids: Benefits and Endpoints in Sport. Nutrients. 2018;11(1):E46.

112. Preston Mason R. New Insights into Mechanisms of Action for Omega-3 Fatty Acids in Atherothrombotic Cardiovascular Disease. Curr Atheroscler Rep. 2019;21(1):2.

113. Du J, Wang T, Huang P, et al. Clinical correlates of decreased plasma coenzyme Q10 levels in patients with multiple system atrophy. Parkinsonism Relat Disord. 2018;57:58-62.

114. Sood B, Keenaghan M. Coenzyme Q10. Treasure Island (FL): StatPearls Publishing; 2018.

115. Zozina VI, Covantev S, Goroshko OA, et al. Coenzyme Q10 in Cardiovascular and Metabolic Diseases: Current State of the Problem. Curr Cardiol Rev. 2018;14(3):164-174.

116. Coenzyme $Q 10$ in the treatment of hypertension: a meta-analysis of the clinical trials. Database of Abstracts of Reviews of Effects (DARE): Quality-assessed Reviews [Internet]. York (UK): Centre for Reviews and Dissemination; 2007.

117. Jafari M, Mousavi SM, Asgharzadeh A, et al. Coenzyme Q10 in the treatment of heart failure: A systematic review of systematic reviews. Indian Heart J. 2018;70 Suppl 1:S111-S117.

118. Ayers J, Cook J, Koenig RA, et al. Recent Developments in the Role of Coenzyme Q10 for Coronary Heart Disease: a Systematic Review. Curr Atheroscler Rep. 2018;20(6):29.

119. Bedoya LM, Bermejo P, Abad MJ. Anti-infectious activity in the Cistaceae family in the Iberian Peninsula. Mini Rev Med Chem. 2009;9(5):519-525.

120. Yue ES, Weidong W, Jie Q. Anti-hyperlipidemia of garlic by reducing the level of total cholesterol and low-density lipoprotein. Medicine (Baltimore). 2018;97(18):e0255.

121. Ried K, Fakler P. Potential of garlic (Allium sativum) in lowering high blood pressure: mechanisms of action and clinical relevance. Integr Blood Press Control. 2014;7:71-82.

122. Maulik SK, Katiyar CK. Terminalia arjuna in cardiovascular diseases: making the transition from traditional to modern medicine in India. Curr Pharm Biotechnol. 2010;11(8):855-860.
123. Dwivedi S, Chopra D. Revisiting Terminalia arjuna-An Ancient Cardiovascular Drug. $\quad J$ Tradit Complement Med. 2014;4(4):224 231.

124. Kaur N, Shafiq N, Negi H, et al. Terminalia arjuna in Chronic Stable Angina: Systematic Review and Meta-Analysis. Cardiology Research and Practice. 2014;2014:281483.

125. Ragini H, Amita P, Jain AK. An approach to standardize Arjunarishta: a well known ayurvedic formulation using UV and Colorimetric method. $J$ Med Pharm Allied Sci. 2012;1:77-84.

126. Shengule SA, Mishra S, Joshi K, et al. Anti-hyperglycemic and antihyperlipidaemic effect of Arjunarishta in highfat fed animals. J Ayurveda Integr Med. 2018;9(1):45-52.

127. Subramaniam S, Subramaniam R, Rajapandian S, et al. AntiAtherogenic Activity of Ethanolic Fraction of Terminalia arjuna Bark on Hypercholesterolemic Rabbits. Evid Based Complement Alternat Med. 2011;2011:487916.

128. Gaikwad D, Jadhav N. A Review on Biogenic Properties of Stem Bark of Terminalia Arjuna: AN UPDATE. Asian J Pharm Clin Res. 2018;11(8):35-39.

129. Kim J, Cha YJ, Lee KH, et al. Effect of onion peel extract supplementation on the lipid profile and antioxidative status of healthy young women: a randomized, placebo-controlled, double-blind, crossover trial. Nutr Res Pract. 2013;7(5):373-379.

130. Yang C, Li L, Yang L, et al. Anti-obesity and Hypolipidemic effects of garlic oil and onion oil in rats fed a high-fat diet. Nutr Metab (Lond). 2018;15:43.

131. Ro JY, Ryu JH, Park HJ, et al. Onion (Allium cepa L.) peel extract has antiplatelet effects in rat platelets. Springerplus. 2015;4:17.

132. Kang YR, Choi HY, Lee JY, et al. Calorie Restriction Effect of HeatProcessed Onion Extract (ONI) Using In Vitro and In Vivo Animal Models. Int J Mol Sci. 2018;19(3):E874.

133. Aslani N, Entezari MH, Askari G, et al. Effect of garlic and lemon juice mixture on lipid profile and some cardiovascular risk factors in people 30-60 years old with moderate hyperlipidaemia: A randomized clinical trial. Int J Prev Med. 2016;7:95.

134. Slimestad R, Fossen T, Vågen IM. Onions: a source of unique dietary flavonoids. J Agric Food Chem. 2007;55(25):10067-10080.

135. Khiari Z, Makris DP. Stability and transformation of major flavonols in onion (Allium cepa) solid wastes. J Food Sci Technol. 2012;49(4):489 494.

136. Kawser Hossain M, Abdal Dayem A, Han J, et al. Molecular Mechanisms of the Anti-Obesity and Anti-Diabetic Properties of Flavonoids. Int J Mol Sci. 2016;17(4):569.

137. Gopa B, Bhatt J, Hemavathi KG. A comparative clinical study of hypolipidemic efficacy of Amla (Emblica officinalis) with 3-hydroxy3-methylglutarylcoenzyme-A reductase inhibitor simvastatin. Indian $J$ Pharmacol. 2012;44(2):238-242.

138. Kanthe PS, Patil BS, Bagali SC, et al. Protective effects of Ethanolic Extract of Emblica officinalis (amla) on Cardiovascular Pathophysiology of Rats, Fed with High Fat Diet. J Clin Diagn Res. 2017;11(9):CC05CC09.

139. Khanna S, Das A, Spieldenner J, et al. Supplementation of a standardized extract from Phyllanthus emblica improves cardiovascular risk factors and platelet aggregation in overweight/class-1 obese adults. J Med Food. 2015;18(4):415-420

140. Tamang HK, Timilsina U, Singh KP, et al. Apo B/Apo A-I Ratio is Statistically A Better Predictor of Cardiovascular Disease (CVD) than Conventional Lipid Profile: A Study from Kathmandu Valley, Nepal. $J$ Clin Diagn Res. 2014;8(2):34-36. 
141. Feingold KR, Grunfeld C. Introduction to Lipids and Lipoproteins. In: Feingold KR, Anawalt B, Boyce A, et al., editors. Endotext. South Dartmouth (MA): MDText.com, Inc.; 2000.

142. Choudhary B, Shetty A, Langade DG. Efficacy of Ashwagandha (Withania somnifera [L.] Dunal) in improving cardiorespiratory endurance in healthy athletic adults. Ayu. 2015;36(1):63-68.

143. Chandrasekhar K, Kapoor J, Anishetty S. A prospective, randomized doubleblind, placebo-controlled study of safety and efficacy of a highconcentration fullspectrum extract of ashwagandha root in reducing stress and anxiety in adults. Indian J Psychol Med. 2012;34(3):255-262.

144. Auddy B, Hazra J, Mitra A, et al. A standardized Withania somnifera extract significantly reduces stress-related parameters in chronically stressed humans. Journal of the American Nutraceutical Association. 2008;11:51-57.

145. Cooley K, Szczurko O, Perri D, et al. Naturopathic care for anxiety: a randomized controlled trial ISRCTN78958974. PLoS One. 2009;4(8):e6628.

146. Deshpande A, Irani N, Balakrishnan R. Study protocol and rationale for a prospective, randomized, double-blind, placebo-controlled study to evaluate the effects of Ashwagandha (Withania somnifera) extract on nonrestorative sleep. Medicine (Baltimore). 2018;97(26):e11299.

147. Mishra LC, Singh BB, Dagenais S. Scientific basis for the therapeutic use of Withania somnifera (ashwagandha): a review. Altern Med Rev. 2000;5(4):334-346.

148. Singh N, Nath R, Lata A, et al. Withania somnifera (ashwagandha), a rejuvenating herbal drug which enhances survival during stress (an adaptogen). Int J Crude Drug Res. 1982;20(1):29-35.

149. Lyle N, Bhattacharyya D, Sur TK, et al. Stress modulating antioxidant effect of Nardostachys jatamansi. Indian $J$ Biochem Biophys. 2009;46(1):93-98.

151. Nandha R, Singh H, Moudgill P, et al. A pilot study to clinically evaluate the role of herbomineral compound "Rakatchap Har" in the management of essential hypertension. Ayu. 2011;32(3):329-332.

152. Ali A, Umar D, Farhan M, et al. Effect of Brahmyadi Churna (Brahmi, Shankhapushpi, Jatamansi, Jyotishmati, Vacha, Ashwagandha) and tablet Shilajatu in essential hypertension: An observational study. J Adv Pharm Technol Res. 2015;6(4):148-153.

153. Aisa R, Yu Z, Zhang X, et al. The Effects of Aqueous Extract from Nardostachys chinensis Batalin on Blood Pressure and Cardiac Hypertrophy in Two-Kidney One-Clip Hypertensive Rats. Evid Based Complement Alternat Med. 2017;2017:4031950.

154. Peterson CT, Denniston K, Chopra D. Therapeutic Uses of Triphala in Ayurvedic Medicine. J Altern Complement Med. 2017;23(8):607-614.

155. Aggarwal BB, Prasad S, Reuter S, et al. Identification of novel antiinflammatory agents from Ayurvedic medicine for prevention of chronic diseases: "reverse pharmacology" and "bedside to bench" approach Curr Drug Targets. 2011;12(11):1595-1653.

156. Mashyal P, Bhargav H, Raghuram N. Safety and usefulness of Laghu shankha prakshalana in patients with essential hypertension: A self controlled clinical study. J Ayurveda Integr Med. 2014;5(4):227-235.

157. Mukherjee PK, Rai S, Bhattacharyya S, et al. Clinical study of 'Triphala'-A well knownphytomedicine from India. Ir J Pharmacol Ther. 2006;5(1):51-54.

158. Singh DP, Mani D. Protective effect of Triphala Rasayana against paracetamolinduced hepato-renal toxicity in mice. J Ayurveda Integr Med. 2015;6(3):181-186.

159. Gurjar S, Pal A, Kapur S. Triphala and its constituents ameliorate visceral adiposity from a high-fat diet in mice with diet-induced obesity. Altern Ther Health Med. 2012;18(6):38-45.
160. Walden R, Tomlinson B. Cardiovascular disease. In: Benzie IFF, Wachtel-Galor S, editors. Herbal medicine: biomolecular and clinical aspects. Boca Raton: CRC Press/Taylor \& Francis; 2011.

161. Tarasiuk A, Mosińska P, Fichna J. Triphala: current applications and new perspectives on the treatment of functional gastrointestinal disorders. Chin Med. 2018;13:39.

162. Saravanan S, Srikumar R, Manikandan S, et al. Hypolipidemic effect of triphala in experimentally induced hypercholesteremic rats. Yakugaku Zasshi. 2007;127(2):385-388.

163. Vinayakumar A. Ayurvedic Clinical Medicine. $1^{\text {st }}$ ed. Delhi: Sri Satguru publication; 1997.

164. A P, P SR, M PR, K G R. Apoptosis in angiotensin II-stimulated hypertrophic cardiac cells -modulation by phenolics rich extract of Boerhavia diffusa L. Biomed Pharmacother. 2018;108:1097-1104.

165. Prathapan A, Vineetha VP, Abhilash PA, et al. Boerhaavia diffusa L. attenuates angiotensin II-induced hypertrophy in $\mathrm{H} 9 \mathrm{c} 2$ cardiac myoblast cells via modulating oxidative stress and down-regulating NF- $\mathrm{\kappa} \beta$ and transforming growth factor $\beta 1$. Br J Nutr. 2013;110(7):1201-1210.

166. Ferreres F, Sousa C, Justin M, et al. Characterisation of the phenolic profile of Boerhaavia diffusa L. by HPLC-PAD-MS/MS as a tool for quality control. Phytochem Anal. 2005;16(6):451-458.

167. Yan L, Zhang JD, Wang B, et al. Quercetin inhibits left ventricular hypertrophy in spontaneously hypertensive rats and inhibits angiotensin II-induced $\mathrm{H} 9 \mathrm{C} 2$ cells hypertrophy by enhancing PPAR $\gamma$ expression and suppressing AP-1 activity. PLoS One. 2013;8(9):e72548.

168. Larson AJ, Symons JD, Jalili T. Therapeutic potential of quercetin to decrease blood pressure: review of efficacy and mechanisms. Adv Nutr. 2012;3(1):39-46.

169. Liobikas J, Majiene D, Trumbeckaite S, et al. Uncoupling and antioxidant effects of ursolic acid in isolated rat heart mitochondria. $J$ Nat Prod 2011;74(7):1640-1644.

170. Calderón-Montaño JM, Burgos-Morón E, Pérez-Guerrero C, et al. A review on the dietary flavonoid kaempferol. Mini Rev Med Chem. 2011;11(4):298-344.

171. Aviello G, Canadanovic-Brunet JM, Milic N, et al. Potent antioxidant and genoprotective effects of boeravinone $\mathrm{G}$, a rotenoid isolated from Boerhaavia diffusa. PLoS One. 2011;6(5):e19628.

172. Lami N, Kadota S, Kikuchi T, et al. Constituents of the roots of Boerhaavia diffusa L. III. Identification of $\mathrm{Ca} 2+$ channel antagonistic compound from the methanol extract. Chem Pharm Bull (Tokyo). 1991;39(6):1551-1555

173. Vineetha VP, Prathapan A, Soumya RS, et al. Arsenic trioxide toxicity in $\mathrm{H} 9 \mathrm{c} 2$ myoblasts-damage to cell organelles and possible amelioration with Boerhavia diffusa. Cardiovasc Toxicol. 2013;13(2):123-137.

174. Hung HC, Joshipura KJ, Jiang R, et al. Fruit and vegetable intake and risk of major chronic disease. J Natl Cancer Inst. 2004;96(21):1577-1584.

175. Wiseman M. The second World Cancer Research Fund/American Institute for Cancer Research expert report. Food, nutrition, physical activity, and the prevention of cancer: a global perspective. Proc Nutr Soc. 2008;67(3):253-256.

176. 10 Incredible Reasons to Eat Cruciferous Vegetables Regularly. Web Food Revolution Network; 2017.

177. Bai $Y$, Wang X, Zhao S, et al. Sulforaphane Protects against Cardiovascular Disease via Nrf2 Activation. Oxid Med Cell Longev. 2015;2015:407580.

178. Choi KM, Lee YS, Kim W, et al. Sulforaphane attenuates obesity by inhibiting adipogenesis and activating the AMPK pathway in obese mice. J Nutr Biochem. 2014;25(2):201-207. 
179. Health Dangers of Cruciferous Vegetables. Web Kevin Stock; 2018

180. Mohtashami A, Entezari MH. Effects of Nigella sativa supplementation on blood parameters and anthropometric indices in adults: A systematic review on clinical trials. $J$ Res Med Sci. 2016;21:3.

181. Jaarin K, Foong WD, Yeoh MH, et al. Mechanisms of the antihypertensive effects of Nigella sativa oil in L-NAME-induced hypertensive rats. Clinics (Sao Paulo). 2015;70(11):751-757.

182. Enayatfard L, Mohebbati R, Niazmand S, et al. The standardized extract of Nigella sativa and its major ingredient, thymoquinone, ameliorates angiotensin II-induced hypertension in rats. J Basic Clin Physiol Pharmacol. 2018;30(1):51-58.

183. Nagi MN, Al-Shabanah OA, Hafez MM, et al. Thymoquinone supplementation attenuates cyclophosphamide-induced cardiotoxicity in rats. J Biochem Mol Toxicol. 2011;25(3):135-142.

184. Ebru U, Burak U, Yusuf S, et al. Cardioprotective effects of Nigella sativa oil on cyclosporine A-induced cardiotoxicity in rats. Basic Clin Pharmacol Toxicol. 2008;103(6):574-580.

185. Al-Shabanah OA, Badary OA, Nagi MN, et al. Thymoquinone protects against doxorubicin-induced cardiotoxicity without compromising its antitumor activity. J Exp Clin Cancer Res. 1998;17(2):193-198.
186. Torabi M, Naeemzadeh F, Ebrahimi V, et al. The Effect Of Zingiber Officinale (Ginger) On Hypertension; A Systematic Review Of Randomised Controlled Trials. BMJ Open. 2017;7(Suppl 1):48-49.

187. Brahma Naidu P, Uddandrao VV, Ravindar Naik R, et al. Ameliorative potential of gingerol: Promising modulation of inflammatory factors and lipid marker enzymes expressions in HFD induced obesity in rats. Mol Cell Endocrinol. 2016;419:139-147.

188. Zhu J, Chen H, Song Z, et al. Effects of Ginger (Zingiber officinale Roscoe) on Type 2 Diabetes Mellitus and Components of the Metabolic Syndrome: A Systematic Review and Meta-Analysis of Randomized Controlled Trials. Evid Based Complement Alternat Med. 2018;2018:5692962.

189. Li X, Guo J, Liang N, et al. 6-Gingerol Regulates Hepatic Cholesterol Metabolism by Up-regulation of LDLR and Cholesterol Efflux-Related Genes in HepG2 Cells. Front Pharmacol. 2018;9:159.

190. Tabibi H, Imani H, Atabak S, et al. Effects of Ginger on Serum Lipids and Lipoproteins in Peritoneal Dialysis Patients: A Randomized Controlled Trial. Perit Dial Int. 2016;36(2):140-145.

191. Ali AMA, El-Nour MEM, Yagi SM. Total phenolic and flavonoid contents and antioxidant activity of ginger (Zingiber officinale Rosc.) rhizome, callus and callus treated with some elicitors. J Genet Eng Biotechnol. 2018;16(2):677-682. 